\title{
REFLECTION OF NONLINEAR DEEP-WATER WAVES INCIDENT ONTO A WEDGE OF ARBITRARY ANGLE
}

\author{
T. R. MARCHANT ${ }^{1}$ AND A. J. ROBERTS ${ }^{2}$
}

(Received 12 September 1988; revised 6 March 1989)

\begin{abstract}
Wave reflection by a wedge in deep water is examined, where the wedge can represent a breakwater of finite length or the bow of a ship heading directly into the waves. In addition, the form of the solution allows the results to apply to ships heading at an angle into the waves. We consider a deep-water wavetrain approaching the wedge head on from infinity and being reflected. Far from the wedge there is a field of progressive waves (the incident wavetrain) while close to the wedge there is a short-crested wavefield (the incident and reflected wavetrains). A weakly-nonlinear slowly-varying averaged Lagrangian theory is used to describe the problem (see Whitham [16]) as the theory includes the nonlinear interaction between the incident and reflected wavetrains. This modelling of a short-crested wavefield allows the nonlinear wavefield to be found for broad wedges, as opposed to previous theories which are applicable to thin wedges only.

It is shown that the governing partial differential equations are hyperbolic and that the solution comprises two regions, within which the wave properties are constant separated by a wave jump. Given the wedge angle and the incident wavefield, the jump angle and the wave steepness and wavenumber of the short-crested wavefield behind the wave jump can be determined. Two solution branches are found to exist: one corresponds to regular reflection, while for small amplitudes the other is similar to Mach-reflection and so it is called near Mach-reflection. Results are presented describing both solution branches and the transition between them.
\end{abstract}

\section{Introduction}

Wave reflection and diffraction by a wedge in water is of interest in practical situations such as for a breakwater of finite length or the bow of a ship heading directly or at an angle into the waves.

\footnotetext{
'Department of Mathematics, University of Wollongong, N.S.W. 2500, Australia.

${ }^{2}$ Department of Applied Mathematics, University of Adelaide, S.A. 5000, Australia.

(C) Copyright Australian Mathematical Society 1990, Serial-fee code 0334-2700/90
} 
In the linear analysis, a wavetrain approaches the wedge head on from infinity and is reflected by the wedge, the incident and reflected waves on either side of the wedge being symmetric about the boundary's normal. However, this picture can be significantly modified for nonlinear waves. Experimental observations for waves of glancing incidence upon a wall in water of shallow depth (Perroud [11], Chen [1] and Wiegel [17]) show that the reflection is not regular. Instead, a wave develops which propagates along the wall (called a Mach-stem). This phenomenon is called Mach-reflection due to its similarity to the reflection of a shock wave in supersonic flow. As the incident wave becomes less oblique, the Mach-stem disappears and the wave reflection is qualitatively similar to that of linear wavetrains.

Much progress has been made on the problem of the forward scattering or diffraction of a wavetrain striking a thin wedge at glancing incidence. A parabolic assumption is made where the variation in the wave amplitude in the direction of propagation is small $\left(0\left(\epsilon^{2}\right)\right.$, where $\epsilon$ is the order of the wave amplitude) compared with the spanwise variation (which is $0(\epsilon)$ ). This approximation was used by Mei and Tuck [8] and Tsay and Liu [14] in deriving the linear parabolic wave equation which describes the wave envelope. Mei and Tuck [8] obtained numerical results for a thin submerged obstacle head on to the waves in water of otherwise constant depth. Their results show that a significant amplification of the wave amplitude occurs in the vicinity of the obstacle. Tsay and Liu [14] derived a similar linear equation valid for mildly sloping sea beds. They considered two problems: the diffraction of obliquely incident waves in water of slowly varying depth, and the wavefield in the region of a submerged circular shoal. It was shown that increased wave amplitudes occur in both examples. However, in the region of large wave amplitudes a linear theory is not valid so a weakly-nonlinear theory must be considered.

Yue and Mei [18] consider a weakly-nonlinear theory to study the diffraction of waves in water of finite depth by a thin wedge. Again using the parabolic approximation, they derived a nonlinear Schrödinger equation which describes the wave envelope. They give extensive numerical solutions which show that a Mach-stem develops as the incident wave amplitude is increased. Outside the Mach-stem region fluctuations of smaller amplitude join the Mach-stem with the incident wavefield. Also, as the wave propagates along the wedge the transition region spreads out linearly. In addition they show that by ignoring the fluctuations, the Mach-stem can be approximated as a constant wavefield, separated by a wave jump, or shock, from the incident wavefield. They obtain good agreement with their numerical solutions by this approximation. 
Kirby and Dalrymple [4] derived a nonlinear Schrödinger equation in a similar way to Yue and Mei [18] but allowed for slow depth variation. They considered two numerical examples: a submerged circular shoal, and a wedge shaped depression. For the case of the submerged circular shoal their results show that linear theory substantially overpredicts the wave amplitude in the vicinity of the shoal. In addition, wave jumps can be identified and are qualitatively similar to Yue and Mei's results. For the case of the wedge shaped depression, linear and weakly-nonlinear theory are again compared. The linear results show the presence of a caustic along the line of the wedge depression. The wave amplitude is small inside the depression region; hence this indicates that the wave is almost totally reflected (see their Figure 3 ). In the far-field (when the wave has propagated a long way along the wedge) the wavefield can be described by an Airy function solution. The corresponding weakly-nonlinear far-field solution is described in terms of the second Painlevé transcendent. The weakly-nonlinear solutions show that for large incident wave amplitudes, the linear picture changes and a broad region of progressive waves with slowly varying amplitude occurs along the depression line, and extends well inside the depression region. This wave can be identified as a Mach-stem. Hence for large wave amplitudes the far-field solution involving the second Painlevé transcendent is unlikely to occur, as Machreflection occurs instead, with very little or no reflected wave.

Peregrine [9] considered the reflection, refraction and diffraction of finiteamplitude water waves associated with wave jumps. First, he examined the problem of Mach-reflection, which can be simplified to the consideration of a single wavetrain in deep water incident upon a wedge with the wavetrain constrained to travel parallel to the wedge after the wave jump. To locate the wave jump he uses conservation of wave action, conservation of wavenumber along the jump and conservation of frequency (see his Figure 4). Numerically exact integral properties of finite-amplitude progressive waves are used in the calculations. For small wedge angles and low waves his solutions compare well with the wave jump approximation of Yue and Mei [18]. This extension to finite-amplitude waves has the disadvantage that the structure of the wave jump is lost (the structure of Yue and Mei's wave jump is the region of fluctuating wave amplitude between the Mach-stem and the incident wavefield; see their Figures 3, 4 and 6). Secondly, Peregrine noticed that the wave jump found by Yue and Mei looks like an undular bore propagating away from the wedge. He takes this analogy further by transforming the nonlinear Schrödinger equation into a form equivalent to the shallow water equations used to describe undular or turbulent bores. Thirdly, he considered the defocusing nature of the nonlinear Schrödinger equation for gravity water 
waves. Using an hydraulic analogy he examined the effect that nonlinearity has upon a linear caustic. Linear theory results in a cusp caustic, while the weakly-nonlinear solution defocuses this cusp caustic into two separate wave jumps or caustic lines (see his Figure 5). Hence the conclusion from this and from the results of Yue and Mei [18] and Kirby and Dalrymple [4] is that nonlinear wave jumps are not necessarily similar to linear wave jumps; there may be qualitative differences.

Peregrine ([10], Section 6) considers the dark soliton solution of the nonlinear Schrödinger equation, so-called because it results in the wave amplitude decreasing below the amplitude of the carrier wavetrain. His Figure 8 (a) shows these solitons developing on a smooth wave jump between two uniform wavefields. The $x$ and $t$ directions there correspond respectively to the spanwise and wave propagation directions in Yue and Mei's wedge geometry. The similarity with Yue and Mei's solutions is readily apparent (see their Figure 4).

Whitham [15] considers nonlinear dispersive waves via an averaged Lagrangian approach. The variational equations form a system of nonlinear hyperbolic partial differential equations which can lead to multi-valued solutions. However, the slowly-varying wave can be saved by the inclusion of a wave jump (as given by the Rankine-Hugoniot conditions) in the region of the multiple solutions. In contrast, Yue and Mei [18] and Kirby and Dalrymple [4] derived a nonlinear Schrödinger equation, which because of the resolution of shorter length scales, describes the solution in the transition region near the wave jump without becoming invalid (as in Marchant and Roberts [7] where the nonlinear ray theory became invalid at a circular caustic while a modified nonlinear Schrödinger equation provides a valid description of the wavefield in the caustic region). Slowly-varying wave theory is used in another context by Howard and Kopell [3]. They consider slowly-varying solutions to reaction-diffusion equations and find shocks or wave jumps occuring. Again, consideration of the full equations leads to information about the shock region, such as its thickness (see their Section 4).

In Section 2 we consider the problem of a deep-water wavetrain directly approaching a wedge from infinity, and then being reflected (however, the form of the solutions allow the results to apply to wavetrains approaching the wedge at an angle). Far from the wedge there is a field of progressive waves (the incident wavetrain) while close to the wedge there is a field of short-crested waves (the incident and reflected wavetrains). Thus the problem is formulated, as in Marchant and Roberts [7], by using the slowly-varying averaged Lagrangian theory of Whitham [16] which includes the nonlinear interaction between the incident and reflected wavetrains. This is in contrast to both Peregrine [9] and Yue and Mei [18] who considered only a single wavetrain, not allowing for reflection at the wedge. Thus the consideration of the 
short-crested wavefield allows reflection from broad wedges to be examined. This research complements Marchant and Roberts [7] where the wavefield considered varies in the radial direction but has no angular variation; here the wavefield varies in the angular direction but has no radial variation.

In Section 3 the linear solution is derived by the method of characteristics, the governing partial differential equations being hyperbolic. In Section 4 a limit involving small wedge angle and small amplitudes is considered. The governing partial differential equations are hyperbolic and, by the method of characteristics, the solution is shown to be two constant wave regions separated by a wave jump. Hence in Section 5 a numerical scheme is developed for arbitrary wedge angle and wave steepness. We consider the problem as a wavefield of constant progressive waves separated by a wave jump from a wavefield of short-crested waves. The variational principle gives equations comprising both algebraic and partial differential equations. The partial differential equations provide the Rankine-Hugoniot conditions to be satisfied across the wave jump. Thus, given the wedge angle and the incident wavefield, the jump angle and the wave steepness and wavenumber of the short-crested wavefield behind the wave jump can be determined. Section 6 considers Mach reflection where there is no reflected wavetrain. Here the incident wavetrain bends through the wave jump to travel parallel to the wedge. This is the situation considered by Peregrine [9] and Yue and Mei [18], and so the results are compared with theirs. In Section 7 results are presented for a wavefield where reflection from the wedge occurs and thus a reflected wavetrain exists and a short-crested wavefield is generated. For a blunt wedge the reflection is regular (where the picture is qualitatively similar to the reflection of linear waves). However, for thin wedges two branches of the solution exist: one corresponding to regular reflection, while for small amplitudes the other is similar to Mach-reflection and so is called near Mach-reflection.

\section{Variational formulation and boundary conditions}

\subsection{The variational equations for a steady wavefield}

We shall describe the interaction of two nonlinear slowly-varying deepwater wavetrains in the same way as Marchant and Roberts [7]. To accomplish this, Whitham's [16] variational formulation is used. This formulation includes a local averaging which removes the oscillations of the wave motion from the equations, leaving the slow variations in space and time of amplitude, wavenumber and frequency to be found. The averaged Lagrangian is obtained by substituting expressions for the wave motion into the Lagrangian and then averaging. The equations obtained from the averaged Lagrangian by 
varying the parameters in the expression then describe the slow variations of the wave properties. We consider a co-ordinate system where $x, y$ are horizontal co-ordinates and the $z$-axis is vertically up. Upon assuming that the fluid is incompressible and inviscid and that the fluid motion is irrotational, we consider a velocity potential $\phi$ and a free surface shape $\eta$ describing the two wavetrains given by

$$
\phi-\phi\left(\mathbf{Q}_{1}, \mathbf{Q}_{2}, z\right), \quad \eta=\eta\left(\Theta_{1}, \Theta_{2}\right),
$$

where $\Theta_{1}(x, y, t)$ and $\Theta_{2}(x, y, t)$ are two phase functions. Thus $\phi$ and $\eta$ are periodic in both $\Theta_{1}$ and $\Theta_{2}$ with period $2 \pi$ and

$$
\mathbf{k}_{j}=\nabla \Theta_{j}, \quad \omega_{j}=-\frac{\partial \Theta_{j}}{\partial t}, \quad j=1,2,
$$

where $\mathbf{k}_{j}$ are the wavenumber vectors, and $\omega_{j}$ are the frequencies of the two phases. For simplicity only deep water is considered, for which the Lagrangian, as proposed by Luke [5] is

$$
L=-\rho \int_{-\infty}^{\eta}\left(\phi_{t}+\frac{1}{2}|\nabla \phi|^{2}\right) d z-\frac{\rho g}{2} \eta^{2},
$$

where $\rho$ is the density of water and $g$ is the gravitational acceleration. The variational principle for water waves is one of stationary pressure; (2.3) just represents the depth integrated pressure of the fluid. We write $\phi$ and $\eta$ in the following truncated form

$$
\begin{aligned}
\phi= & b_{1} e^{k_{1} z} \sin \left(\Theta_{1}\right)+b_{2} e^{k_{2} z} \sin \left(\Theta_{2}\right) \\
& +b_{3} e^{2 k_{1} z} \sin \left(2 \Theta_{1}\right)+b_{4} e^{2 k_{2} z} \sin \left(2 \Theta_{2}\right) \\
& +b_{5} e^{\left|\mathbf{k}_{1}+\mathbf{k}_{2}\right| z} \sin \left(\Theta_{1}+\Theta_{2}\right)+b_{6} e^{\left|\mathbf{k}_{1}-\mathbf{k}_{2}\right| z} \sin \left(\Theta_{1}-\Theta_{2}\right),
\end{aligned}
$$

and

$$
\begin{aligned}
\eta= & a_{1} \cos \left(\Theta_{1}\right)+a_{2} \cos \left(\Theta_{2}\right)+a_{3} \cos \left(2 \Theta_{1}\right)+a_{4} \cos \left(2 \Theta_{2}\right) \\
& +a_{5} \cos \left(\Theta_{1}+\Theta_{2}\right)+a_{6} \cos \left(\Theta_{1}-\Theta_{2}\right) .
\end{aligned}
$$

Normally, $b_{1}, b_{2}, a_{1}$ and $a_{2}$ will be of first order in wave amplitude while the other coefficients will be of second order. The largest neglected higher harmonics in these expressions will be of third order; thus this analysis is valid only for waves which are not too high.

Upon substituting the above expressions into (2.3) and using (2.2), an expression is found for

$$
L=L\left(a_{i}, b_{i}, \omega_{j}, \mathbf{k}_{j}, \boldsymbol{\Theta}_{j}\right)
$$

Assuming $a_{i}, b_{i}, \omega_{j}$ and $\mathbf{k}_{j}$ to be slowly-varying functions of space and time and thus effectively constant over a wave period, we define the averaged 
Lagrangian to be

$$
\bar{L}\left(a_{i}, b_{i}, \omega_{j}, \mathbf{k}_{j}\right)=\frac{1}{(2 \pi)^{2}} \int_{0}^{2 \pi} \int_{0}^{2 \pi} L d \Theta_{1} d \Theta_{2} .
$$

$\bar{L}$ is truncated to fourth order, as the neglected harmonics first contribute at sixth order. $\bar{L}$ contains the information about the interaction of the slow variations in amplitude, wavenumber and frequency of the two wavetrains. According to Whitham [15], the variational equations to be solved are then

$$
\left.\begin{array}{c}
\left.\begin{array}{c}
\bar{L}_{a_{i}}=0 \\
\bar{L}_{b_{i}}=0
\end{array}\right\} \quad i=1, \ldots 6, \\
\nabla \times \mathbf{k}_{j}=\mathbf{0} \\
\partial \mathbf{k}_{j} / \partial t+\nabla \omega_{j}=\mathbf{0} \\
\partial \bar{L}_{\omega_{j}} / \partial t-\nabla \cdot \bar{L}_{\mathbf{k}_{j}}=0
\end{array}\right\} \quad j=1,2 .
$$

Equations (2.8) are the results of variations of $\bar{L}$ with respect to the independent amplitude variables. The last of the equations (2.9) is the result of variations with respect to the independent phase function $\Theta_{j}$. The remaining equations in (2.9) are conditions on $\mathbf{k}_{j}$ and $\omega_{j}$ which ensure that $\Theta_{j}$ exists.

In particular, for wavefields where all the properties are steady $(\partial / \partial t=0)$ (2.8) and (2.9) become

$$
\left.\begin{array}{l}
\bar{L}_{a_{i}}=0 \\
\bar{L}_{b_{i}}=0
\end{array}\right\} \quad i=1, \ldots 6
$$

and

$$
\left.\begin{array}{l}
\nabla \times \mathbf{k}_{j}=\mathbf{0} \\
\omega_{j}=\text { constant } \\
\nabla \cdot \bar{L}_{\mathbf{k}_{j}}=0
\end{array}\right\} j=1,2 .
$$

\subsection{Initial and boundary conditions}

For a wedge of apex angle $2 \alpha$ (see Figure 1) $(x, y)$ co-ordinates are used as shown. Firstly the wavenumber of the incident wavetrain is defined by

$$
\left.\begin{array}{l}
\mathbf{k}_{1}=\cos (\alpha) \mathbf{i}-\sin (\alpha) \mathbf{j} \\
\omega_{1}=\omega
\end{array}\right\} \quad \text { as } x \cos (\alpha)-y \sin (\alpha) \rightarrow-\infty .
$$

This just defines the incident wavetrain to be directly incident upon the wedge with $k_{1}$ normalised to 1 . In addition the gravitational acceleration $g$ is normalised to 1 . Hence, for the linear case $\omega_{1}=1$, while for the nonlinear 


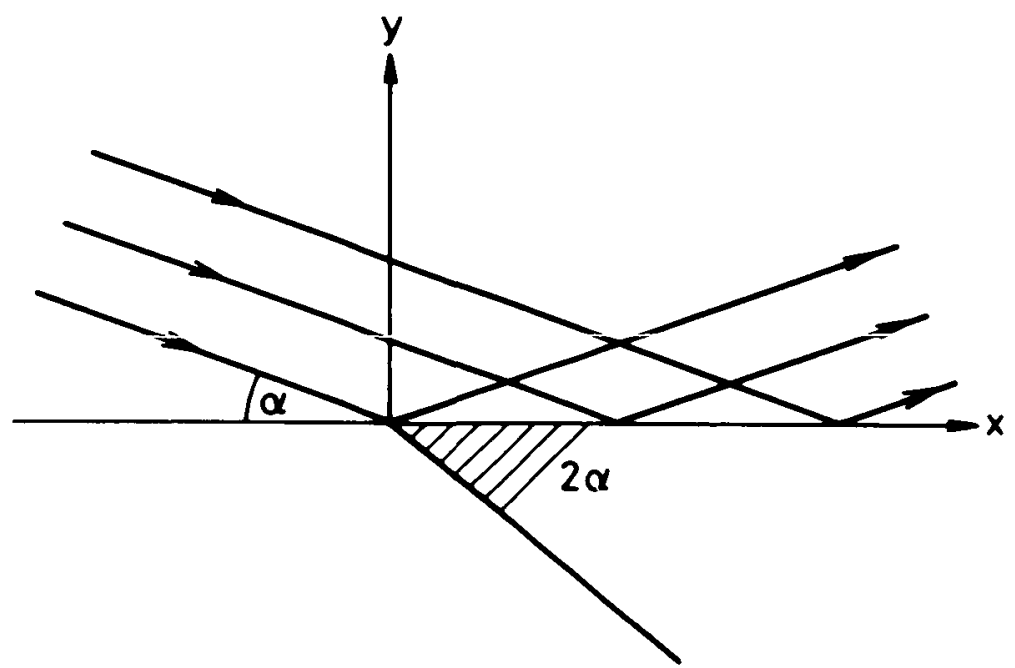

FIGURE 1. Definition sketch of the problem. Drawn is a wedge with apex angle $2 \alpha$ with the positive $x$ axis forming the wedge boundary. The incident wavetrain travels at an angle $\alpha$ to the negative $x$ axis and is reflected at the wedge.

case setting $\omega_{1}$ to a constant value $\omega$ implicitly fixes the amplitude of the incident wavefield via the nonlinear dispersion relation $\left(\mathbf{k}_{1}\right.$ and $g$ remain 1 in the nonlinear case).

Also the wedge boundary must be impervious, that is $\partial \phi / \partial y=0$ on $y=0$. Differentiating (2.4) and neglecting terms involving $a_{i}^{\prime}$ and $k_{j}^{\prime}$ since they are negligible in the slowly-varying approximation we get

$$
\begin{aligned}
\frac{\partial \phi}{\partial y}= & k_{1 y} b_{1} e^{k_{1} z} \cos \left(\Theta_{1}\right)+k_{2 y} e^{k_{2} z} \cos \left(\Theta_{2}\right) \\
& +2 k_{1 y} b_{3} e^{2 k_{1} z} \cos \left(2 \Theta_{1}\right)+2 k_{2 y} b_{4} e^{2 k_{2} z} \cos \left(2 \Theta_{2}\right) \\
& +\left(k_{1 y}+k_{2 y}\right) b_{5} e^{\left|\mathbf{k}_{1}+k_{2}\right| z} \cos \left(\Theta_{1}+\Theta_{2}\right) \\
& +\left(k_{1 y}-k_{2 y}\right) b_{6} e^{\left|k_{1}-k_{2}\right| z} \cos \left(\Theta_{1}-\Theta_{2}\right) .
\end{aligned}
$$

The terms of (2.13) can only only cancel if $\Theta_{1}=\Theta_{2}$ and $k_{1}=k_{2}$. Thus (2.13) gives, as the boundary condition on the wedge,

$$
\left.\begin{array}{l}
k_{1 x}=k_{2 x}, \quad k_{1 y}=-k_{2 y}, \\
b_{1}=b_{2}, \quad b_{3}=b_{4}, \quad b_{6}=0 .
\end{array}\right\} \quad \text { on } y=0 .
$$

Another alternative for the boundary condition is to let $a_{2}=0$ (no reflected wavetrain) and $k_{1 y}=0$ on $y=0$. This alternative corresponds to Mach-reflection where the wavefield is composed of two regions of progressive wavefields separated by a wave jump (one progressive wavefield is the incident wave while the other behind the wave jump is constrained to travel 
parallel to the wedge). This is the case investigated by Peregrine [9] and Yue and Mei [18].

\section{The linear solution}

Initially the linear solution is considered (obtained by retaining only the lowest order terms in the Lagrangian) to illustrate the nature of the solution. To lowest order,

$$
\left.\begin{array}{ll}
\bar{L}_{a_{j}}=-2 \omega b_{j}+2 k_{j} a_{j}, & \bar{L}_{b_{j}}=-2 \omega a_{j}+2 k_{j} b_{j} \\
\bar{L}_{k_{j x}}=k_{j x} b_{j}^{2}, & \bar{L}_{k_{j y}}=k_{j y} b_{j}^{2}
\end{array}\right\}, \quad j=1,2 .
$$

Combining the first two equations of (3.1) and letting $\omega=1$ gives

$$
k_{j}^{2}=1 \text { and } a_{j}=b_{j}, \quad j=1,2 .
$$

Substituting for $k_{j y}$ in the first of (2.11) gives

$$
\frac{\partial k_{j x}}{\partial x}+\left(\frac{k_{j y}}{k_{j x}}\right) \frac{\partial k_{j x}}{\partial y}=0, \quad j=1,2 .
$$

The solution to this partial differential equation is

$$
d k_{j x} / d x=0 \quad \text { on } d y / d x=k_{j y} / k_{j x}, \quad j=1,2 .
$$

Hence $k_{j y}$ and $k_{j x}$ are constant along their characteristic directions. Now the last of (2.2) is considered along the characteristics of (3.3). This equation becomes

$$
\frac{\partial b_{j}}{\partial x}+\left(\frac{k_{j y}}{k_{j x}}\right) \frac{\partial b_{j}}{\partial y}=0, \quad j=1,2 .
$$

Hence $b_{j}$ is constant along these same characteristics. The initial conditions give the solution along the characteristics which correspond to the initial wavetrain,

$$
k_{1 x}=\cos (\alpha), \quad k_{1 y}=-\sin (\alpha), \quad a_{1}=b_{1}=a_{1} \quad \text { on } y=-\tan (\alpha) x+c,
$$

where $a_{I}$ is the amplitude of the incident progressive wavefield. The boundary conditions give the solution along the characteristics corresponding to the reflected wavetrain,

$$
k_{2 x}=\cos (\alpha), \quad k_{2 y}=\sin (\alpha), \quad a_{2}=b_{2}=a_{1} \quad \text { on } y=\tan (\alpha) x+c,
$$

for $c \geq 0$. For $c<0, a_{2}=b_{2}=0$ and there is no reflected wavetrain. 
The solution shows the classic ray picture that the incident wavetrain propagates to the wedge in the direction $-\tan (\alpha)$ without change in wave steepness. The reflected wavetrain propagates from the wedge in the direction $\tan (\alpha)$ without change in wave steepness also. There is a jump in the reflected wave steepness from 0 to $a_{I}$ along the characteristic through the wedge tip, $y=\tan (\alpha) x$. The full solution of the linear wave equation would of course describe the transition between the incident progressive wavefie!d and the short-crested wavefield. The linear ray solution, and the later nonlinear ray solution neglects diffractive and refractive effects which would provide details of the structure of the solution in the wave jump region. For example, the linear parabolic wave equation (valid for small wedge angles and derived using the parabolic assumption) includes some of these effects with the far-field solution being described in terms of an Airy function.

\section{Approximate nonlinear solutions involving small amplitudes and small wedge angle}

\subsection{One-phase solutions}

In the nonlinear case the full equations are too complicated for an analytic solution to be obtained. However, by letting both the wedge angle and the wave amplitude be small, that is

$$
\alpha, a_{1} \ll 1,
$$

then the nonlinear equations are amenable to analysis. For this one-phase Mach-reflection case the leading approximations are

$$
\omega^{2} \sim 1+a_{1}^{2}, \quad b_{1} \sim a_{1}, \quad a_{3} \sim a_{1}^{2} / 2, \quad k \sim \omega^{2}-a_{1}^{2}, \quad b_{3} \sim 0,
$$

where $a_{l}$ is the amplitude of the incident progressive wavefield. Equations (2.10) and (2.11) become to leading order

$$
\begin{gathered}
\frac{\partial k_{y}}{\partial x}-\frac{\partial k_{x}}{\partial y}=0, \quad \frac{\partial\left(k_{x} a_{1}^{2}\right)}{\partial x}+\frac{\partial\left(k_{y} a_{1}^{2}\right)}{\partial y}=0, \\
k=\omega^{2}-a_{1}^{2} .
\end{gathered}
$$

By substituting for $k_{y}$ in the two partial differential equations (4.3) a set of two equations in two unknowns of the form

$$
A \mathbf{v}_{x}+B \mathbf{v}_{y}=0 \text {, }
$$

is obtained, where

$$
A=\left(\begin{array}{cc}
k_{x} / k_{y} & 2 a_{1} / k_{y} \\
a_{1}^{2} & 2 k_{x} a_{1}
\end{array}\right), \quad B=\left(\begin{array}{cc}
1 & 0 \\
-\left(k_{x} / k_{y}\right) a_{1}^{2} & 2 k_{y} a_{1}-2 a_{1}^{3} / k_{y}
\end{array}\right),
$$

and $v^{t}=\left(k_{x}, a_{1}\right)$. 
We solve for the eigenvalues $\lambda_{i}$ of the system $(B-\lambda A)$ and the left eigenvectors $\mathrm{l}_{i}$ which satisfy $\mathrm{l}_{i}\left(B-\lambda_{i} A\right)=0$. Then the system of partial differential equations is reduced to the set of ordinary differential equations of the form $1_{i} d v / d x=0$ along the characteristic directions $d y / d x=\lambda_{i}$. To leading order the eigenvalues of the system (4.4) give the characteristics

$$
\frac{d y}{d x}=\frac{k_{y}}{k_{x}} \pm \frac{a_{1}}{k_{x}^{2}} .
$$

The corresponding left eigenvectors are $\mathbf{l}_{i}=\left(\mp a_{1}, 1\right)$ and hence on (4.5)

$$
\frac{d k_{x}}{d x} \mp 2 k_{y} \frac{d a_{1}}{d x}=0,
$$

which can be integrated to

$$
k_{x} \mp 2 k_{y} a_{1}=C_{ \pm},
$$

along the respective characteristics. In the incident wavefield the constants of integration are

$$
C_{ \pm}=\cos (\alpha) \pm 2 \sin (\alpha) a_{I},
$$

along the characteristic directions

$$
d y / d x=-\tan (\alpha) \pm a_{l} .
$$

Hence everywhere in the incident wavefield the wavefield properties will be constant.

Equation (4.9) shows the splitting of the linear characteristic direction into two slightly different directions which is typical of nonlinear waves. This derivation of the small amplitude characteristics is similar to that done by Whitham ([16], Section 15.1) and shows that the splitting is proportional to the wave amplitude.

In the wavefield next to the wedge $k_{y}=0$, hence the last of (4.3) provides an additional equation relating $k_{x}$ and $a_{1}$. Thus there are two equations for the two unknowns along the characteristics and so the wave properties must be constant along

$$
d y / d x= \pm a_{1} .
$$

A family of characteristics from each of the two constant regions will overlap, predicting a multi-valued solution, and hence a discontinuous shock or wave jump must be fitted in. This can be seen in Figure 7 which shows the characteristics for a wavefield corresponding to Mach-reflection. Behind the wave jump one family of characteristics emanate from the wedge while the other family emanate from the wave jump and can be considered as the continuation of one family of characteristics in the incident wavefield. The 
characteristics corresponding to the solid lines can be seen to intersect at the wave jump.

If a wave jump occurs across the line $y=\tan (j) x$ then for a conservation equation of the form

$$
\partial P / \partial x+\partial Q / \partial y=0
$$

the Rankine-Hugoniot jump condition is

$$
\tan (j)[P]=[Q],
$$

where [ ] refers to the difference in the quantity across the wave jump. Applying the jump condition to the two partial differential equations of (4.3) combined with the last of (4.3) gives the three equations

$$
\begin{gathered}
\tan (j) \sin (\alpha)+\left(k_{x}-\cos (\alpha)\right)=0, \\
\tan (j)\left(k_{x} a_{1}^{2}-\cos (\alpha) a_{I}^{2}\right)-\sin (\alpha) a_{I}^{2}=0, \\
k=1+a_{I}^{2}-a_{1}^{2},
\end{gathered}
$$

where $a_{1}$ denotes the constant amplitude behind the wave jump. The leading order solution is then

$$
\begin{gathered}
j \sim\left(\sqrt{\alpha^{2}+16 a_{I}^{2}}-\alpha\right) / 4, \\
a_{I}^{2} \sim \alpha^{2} / 2+a_{I}^{2}+j \alpha .
\end{gathered}
$$

This leading order solution agrees with Yue and Mei's [18] Mach-stem approximation solutions (their equations (4.20) and (4.21); for deep water and small wedge angles their variables are $K=2\left(a_{I} / \alpha\right)^{2}, E_{-}=\left(a_{1} / a_{I}\right)^{2}$ and $\beta=1+j / \alpha)$.

Making the additional scaling $a_{I} \ll \alpha$ gives the leading order approximations

$$
j \sim 2 a_{I}^{2} / \alpha, \quad a_{1} \sim \alpha / \sqrt{2} .
$$

This solution describes the broad details of the Mach-reflection (which can be seen in Figures 3 and 4 for small $a_{l}$ ): that the jump angle increases like the square of the incident wave amplitude and decreases with the wedge angle; also that for very small incident waves the amplitude of the Mach-stem is large, when compared to the incident wave amplitude $a_{I}$, and proportional to the wedge angle $\alpha$.

\subsection{Two-phase solutions: regular reflection}

Now the regular reflection case (where there are two wavetrains present in the wavefield) is considered. Again the wedge angle $\alpha$ and the amplitudes are taken to be small (it is not essential that $\alpha$ be small in this case, however it simplifies the algebra considerably),

$$
\alpha, a_{1}, a_{2} \ll 1 \text {, }
$$


and we perform a similar analysis as in the one-phase case. The leading approximations are

$$
\begin{gathered}
\omega^{2} \sim 1+a_{l}^{2}, \quad b_{1} \sim a_{1}, \quad a_{3} \sim a_{1}^{2} / 2, \quad b_{2} \sim a_{2}, \\
a_{4} \sim a_{2}^{2} / 2, \quad a_{5} \sim a_{1} a_{2}, \quad b_{5}, b_{6}, a_{6}, b_{3} \sim 0, \\
k_{1} \sim \omega^{2}-a_{1}^{2}-2 a_{2}^{2}, \quad k_{2} \sim \omega^{2}-a_{2}^{2}-2 a_{1}^{2} .
\end{gathered}
$$

So (2.10) and (2.11) become to leading order

$$
\left.\begin{array}{l}
\frac{\partial k_{j y}}{\partial x}-\frac{\partial k_{j x}}{\partial y}=0, \\
\frac{\partial\left(k_{j x} a_{j}^{2}\right)}{\partial x}+\frac{\partial\left(k_{j y} a_{j}^{2}\right)}{\partial y}=0, \\
k_{1}=\omega^{2}-a_{1}^{2}-2 a_{2}^{2}, k_{2}=\omega^{2}-a_{2}^{2}-2 a_{1}^{2} .
\end{array}\right\}
$$

By substituting for $k_{j y}$ in the two partial differential equations (4.18) a set of four equations in four unknowns of the form

$$
A \mathbf{v}_{x}+B \mathbf{v}_{y}=0
$$

is obtained, where

$$
\begin{gathered}
A=\left(\begin{array}{cccc}
k_{1 x} / k_{1 y} & 2 a_{1} / k_{1 y} & 0 & 4 a_{2} / k_{1 y} \\
a_{1}^{2} & 2 k_{1 x} a_{1} & 0 & 0 \\
0 & 4 a_{1} / k_{2 y} & k_{2 x} / k_{2 y} & 2 a_{2} / k_{2 y} \\
0 & 0 & a_{2}^{2} & 2 k_{2 x} a_{2}
\end{array}\right) \\
B=\left(\begin{array}{cccc}
1 & 0 & 0 \\
-\left(k_{1 x} / k_{1 y}\right) a_{1}^{2} & 2 k_{1 y} a_{1}-2 a_{1}^{3} / k_{1 y} & 0 & -4 a_{1}^{2} a_{2} / k_{1 y} \\
0 & 0 & 1 & 0 \\
0 & -4 a_{1} a_{2}^{2} / k_{2 y} & -\left(k_{2 x} / k_{2 y}\right) a_{2}^{2} & 2 k_{2 y} a_{2}-2 a_{2}^{3} / k_{2 y}
\end{array}\right)
\end{gathered}
$$
and $\mathbf{v}^{t}=\left(k_{1 x}, a_{1}, k_{2 x}, a_{2}\right)$.

To leading order the eigenvalues of the system (4.19) give the characteristics

$$
\frac{d y}{d x}=\frac{k_{j y}}{k_{j x}} \pm \frac{a_{j}}{k_{j x}^{2}} \quad j=1,2 .
$$

The corresponding left eigenvectors are $\left(\mp a_{1}, 1,0,0\right)$ and $\left(0,0, \mp a_{2}, 1\right)$ and hence on (4.20)

$$
\begin{aligned}
& \frac{d k_{1 x}}{d x} \mp 2 k_{1 y} \frac{d a_{1}}{d x}=0, \\
& \frac{d k_{2 x}}{d x} \mp 2 k_{2 y} \frac{d a_{2}}{d x}=0,
\end{aligned}
$$


which can be integrated to

$$
\begin{aligned}
& k_{1 x} \mp 2 k_{1 y} a_{1}=C_{1 \pm}, \\
& k_{2 x} \mp 2 k_{2 y} a_{2}=C_{2 \pm},
\end{aligned}
$$

along the respective characteristics. Hence to this level of approximation there is no interaction between the incident and reflected wavetrains, the leâding noñlineâ effect beiñg the splititing of the linear characteristic directions into two slightly different directions, unaffected by the other wavetrain. For the incident wavetrain the constants of integration are

$$
C_{1 \pm}=\cos (\alpha) \pm 2 \sin (\alpha) a_{I},
$$

along the characteristics

$$
d y / d x=-\tan (\alpha) \pm a_{l} .
$$

Hence the properties of the incident wavetrain will be constant throughout the wavefield.

The boundary conditions define the reflected wavetrain at the wedge in terms of the incident wave, so for the characteristics corresponding to the reflected wavetrain which begin at the wedge, the constants of integration are

$$
C_{2 \pm}=C_{1 \pm}
$$

along the characteristics

$$
d y / d x=\tan (\alpha) \pm a_{I} .
$$

At all points between the line $y=\left(\tan (\alpha)-a_{l}\right) x$ (which corresponds to the $C_{2-}$ characteristic which starts at the tip of the wedge) and the wedge itself, the intersection of the two families of characteristics corresponding to the reflected wavetrain, which all begin at the wedge (and hence have constants defined by (4.25)) cause the solution to be constant in this region.

Consideration needs to be given to what occurs in the thin angular region (defined say, as the region between the $C_{2+}$ and the $C_{2-}$ characteristics which start at the wedge tip) between the constant region next to the wedge (as described in the preceeding paragraph) and the incident wavefield. A solution where both families of characteristics are varying in this transition region is not possible. The $C_{2+}$ characteristics in this transition region are defined by (4.25) since they all start at the wedge, while the $C_{2-}$ characteristics would start on a line (in the incident wavefield) where $a_{2}=0$ and $k_{2 x}$ is constant, hence all the $C_{2-}$ constants of integration would be the same. This transition region would then be of constant amplitude and wavenumber. Alternatively an expansive fan of the $C_{2-}$ characteristics in this transition region (each one of the fan of $C_{2-}$ characteristics would have $a_{2}$ and $k_{2 x}$ constant along 


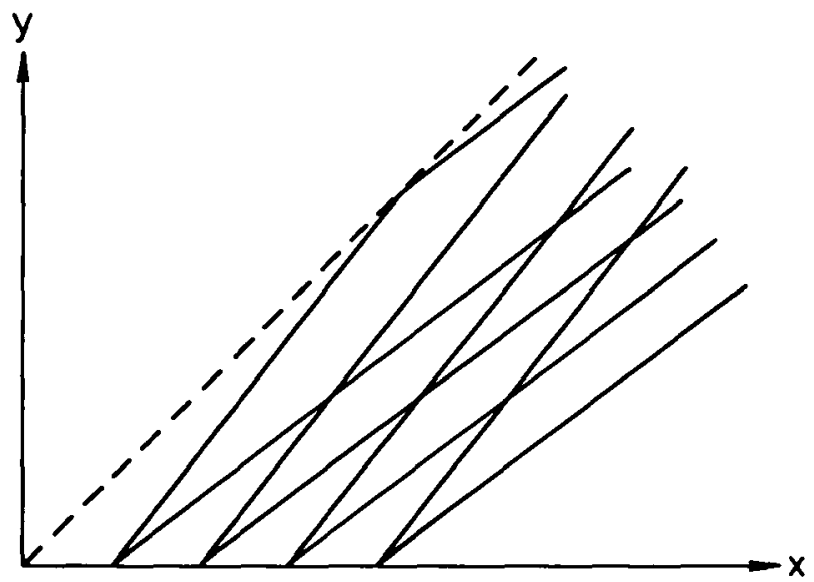

FIGURE 2. The nonlinear characteristics corresponding to the reflected wavetrain which start at the wedge (the positive $x$ axis) have split into two families. The dashed line represents the wave jump.

it) is not possible as the $C_{2-}$ characteristics of the fan would intersect with the $C_{2-}$ characteristics near the wedge. Hence the only feasible solution is a wave jump between the two constant regions of the solution.

Applying the jump conditions (4.12) to the partial differential equations (4.18) gives to leading order

$$
k_{2 x}=\cos (\alpha), \quad k_{2 y}=-\sin (\alpha), \quad a_{2}=a_{l}, \quad j=\alpha .
$$

So to this leading order approximation the wave properties are the same as in the linear solution but are carried along the nonlinear characteristics which have split into two families. Figure 2 shows the splitting of the characteristics corresponding to the reflected wavetrain.

It is shown in Section 7 that actually there exist two solution branches (see Figure 8 for an example) and the solution branch of higher jump angle corresponds to regular reflection. Figures $15(\mathrm{a})$ and $15(\mathrm{~b})$ show the characteristics corresponding to the incident and reflected wavetrains respectively for an example corresponding to the regular reflection.

\subsection{Two-phase solutions: near Mach-reflection}

The solution branch of lower jump angle described in Section 7 correspond to solutions where the wavetrain is glancingly reflected from the wedge. Here a small amplitude and small wedge angle $\alpha$ solution is described corresponding to this lower solution branch. As in Section 4.2 the equations are given to leading order by (4.18) so the characteristics are described by (4.20), (4.21) and (4.22). In Section 4.2 regular reflection was being considered where $k_{1 y}$ 
and $k_{2 y} \sim O(\alpha)$, here glancing reflection is considered so we let

$$
k_{1 y}, k_{2 y} \sim O\left(\alpha^{2}\right),
$$

in the wavefield next to the wedge. Now to leading order the characteristics of the wavefield in this region near the wedge are

$$
d y / d x= \pm a_{j} / k_{j x}^{2} \quad j=1,2 .
$$

These leading order characteristics for the wavefield near the wedge are of the same form as the characteristics for the Mach-reflection solution described in Section 4.1 (see (4.10)). Here though there is an incident and reflected wavetrain near the wedge not just one wavetrain. Using similar arguments as in Section 4.1, a wave jump must be fitted between the incident and reflected wavefields. The characteristics corresponding to the incident wavefield are qualitatively similar to Figure 7 which describes Mach-reflection. For the reflected wavefield one family of characteristics emanate from the wedge while the other family emanates from the wave jump. Hence a close analogy exists between Mach-reflection and near Mach-reflection.

The equations (4.18) and the symmetry of the incident and reflected wavetrains behind the wave jump result in the jump conditions

$$
\begin{gathered}
\tan (j)\left(k_{y}+\sin (\alpha)\right)+\left(k_{x}-\cos (\alpha)\right)=0, \\
\tan (j)\left(k_{x} a_{1}^{2}-\cos (\alpha) a_{l}^{2}\right)-\left(k_{y} a_{1}^{2}+\sin (\alpha) a_{I}^{2}\right)=0, \\
\tan (j) k_{x} a_{1}^{2}+k_{y} a_{1}^{2}=0, \\
k=1+a_{I}^{2}-3 a_{1}^{2} .
\end{gathered}
$$

The solution to $(4.30)$ is

$$
\begin{gathered}
j \sim \frac{a_{I}^{2}}{4 \alpha}-\frac{\alpha}{4}+\frac{1}{4} \sqrt{\alpha^{2}+a_{I}^{4} / \alpha^{2}-10 a_{I}^{2}}, \\
a_{1} \sim \frac{1}{3}\left(\frac{\alpha^{2}}{2}+a_{I}^{2}+j \alpha\right),
\end{gathered}
$$

Making the additional scaling $a_{\jmath} \ll \alpha$ as in Section 4.1, gives the leading order approximations

$$
j \sim 3 a_{I}^{2} / \alpha, \quad a_{1} \sim \alpha / \sqrt{6},
$$

(which can be seen in Figures 8 and 9 for small $a_{1}$ ). This solution differs from the solution (4.15) obtained for Mach-reflection in Section 4.1. Firstly the jump angle is $50 \%$ larger. Secondly, the peak wave steepness behind the jump is $15 \%$ larger (for near Mach-reflection the leading order peak wave steepness is $2 \alpha / \sqrt{6}$ compared with $\alpha / \sqrt{2}$ ) ; however, the mean wave steepness behind 
the wave jump is $26 \%$ smaller (due to the spanwise variation in the wave amplitude of the short-crested wavefield, even though the crests are relatively long). The reasons for these differences are twofold. In near Mach-reflection the wavefield behind the wave jump is relatively long-crested $\left(k_{y} \neq 0\right)$ while for Mach-reflection the wavefield behind the wave jump consists of infinitelycrested progressive waves. Also, as the two-phase wavefield becomes longcrested the dispersion relation becomes

$$
\omega^{2}=k\left(1+3 k^{2} a^{2} / 4\right),
$$

where $a=2 a_{1}$. The coefficient of the nonlinear correction is different from the dispersion relation of progressive waves. This is because for genuinely long-crested waves, the transverse variation of the wavefield is better described in terms of elliptic functions, not trigonometric functions as in this short-crested expansion. Roberts and Peregrine [13] describe this kind of long-crested wavefield. Their solutions provide a smooth variation between the limit of the short-crested expansion (with dispersion relation (4.33)) and the progressive wave expansion. Hence their long-crested wave expansion would provide a more appropriate description for the wavefield next to the wedge for near Mach-reflection than the short-crested expansion used here.

\section{The numerical scheme}

In Section 4 it was shown that a nonlinear model involving small amplitudes and small wedge angle must include a wave jump separating the incident wavefield from the short-crested wavefield near the wedge. The numerical scheme, which is for arbitrary wedge angle $\alpha$, shall use this as its basis by assuming two constant wavefields separated by a wave jump. Also the properties of the wavefield are considered to be steady $(\partial / \partial t=0)$. Far from the wedge only the incident wavetrain is present thus there is a wavefield of progressive waves. Close to the wedge both the incident and reflected wavetrains are present. Hence behind the wave jump a field of short-crested waves is created.

In the region in front of the wave jump we shall define the properties of the wavefield by the subscript $I$. In this region $\mathbf{k}_{I 1}$ is defined by (2.12) and $a_{12}=0$. Due to the boundary conditions the wavenumbers of the shortcrested wavefield are defined by

$$
\mathbf{k}_{1}=k_{x} \mathbf{i}+k_{y} \mathbf{j}, \quad \mathbf{k}_{2}=k_{x} \mathbf{i}-k_{y} \mathbf{j},
$$

and the amplitude coefficients are related by

$$
b_{1}=b_{2}, \quad b_{3}=b_{4}, \quad a_{1}=a_{2}, \quad a_{3}=a_{4} .
$$


The first of the equations in (2.11) represents conservation of wavenumber, the second represents conservation of waves, while the third equation represents conservation of wave-action. According to Peregrine [9] these are precisely the quantities that should be conserved across a wave jump. So by applying the jump conditions (4.12) to the partial differential equations (2.11) and using the symmetry of the short-crested wavefield behind the wave jump ((5.1) and (5.2)) the following jump conditions are obtained,

$$
\left.\begin{array}{l}
\tan (j)\left(k_{y}+\sin (\alpha)\right)+\left(k_{x}-\cos (\alpha)\right)=0 \\
\tan (j)\left(\bar{L}_{k_{x}}-\bar{L}_{k_{l x}}\right)-\left(\bar{L}_{k_{y}}-\bar{L}_{k_{l y}}\right)=0 \\
\tan (j) \bar{L}_{k_{x}}+\bar{L}_{k_{y}}=0 .
\end{array}\right\}
$$

A jump condition is not required for $\nabla \times \mathbf{k}_{2}=0$ since $\mathbf{k}_{2}$ is not defined in front of the wave jump. Also, conservation of waves is required across the wave jump,

$$
\omega_{1}=\omega_{2}=\omega,
$$

where $\omega$ is a constant. In addition to (5.3) and (5.4), (2.10) must be satisfied on both sides of the wave jump,

$$
\left.\begin{array}{llll}
\bar{L}_{a_{11}}=0, & \bar{L}_{a_{l 3}}=0, & \bar{L}_{b_{11}}=0, & \bar{L}_{b_{13}}=0, \\
\bar{L}_{a_{1}}=0, & \bar{L}_{a_{3}}=0, & \bar{L}_{a_{5}}=0, & \bar{L}_{a_{6}}=0, \\
\bar{L}_{b_{1}}=0, & \bar{L}_{b_{3}}=0, & \bar{L}_{b_{5}}=0, & \bar{L}_{b_{6}}=0 .
\end{array}\right\}
$$

(5.3), (5.4) and (5.5) form a set of algebraic equations which can be solved for a given wedge angle $\alpha$ and frequency $\omega$ (which implicitly defines the incident amplitude). Hence $\omega$ remains constant throughout the wavefield while $\mathbf{k}_{1}, \mathbf{k}_{2}$ and the wave amplitude may vary. The equations (the details of which can be found in the Appendix) are too complicated to find an explicit analytic solution so they are solved numerically using a version of Newton's method.

\section{Mach-reflection}

In this section we consider the numerical solution when $\eta=\eta\left(\Theta_{1}\right)$ and $\phi=\phi\left(\Theta_{1}\right)$ are functions of one phase function only. Behind the wave jump the wavetrain must propagate parallel to the wedge, hence the wavefield will consist only of progressive waves. This is Mach-reflection and has been considered by Peregrine [9] and Yue and Mei [18]. Peregrine's solutions are valid for finite-amplitude waves (due to the use of a numerically exact Lagrangian) and hence are more accurate than this weakly-nonlinear model. 


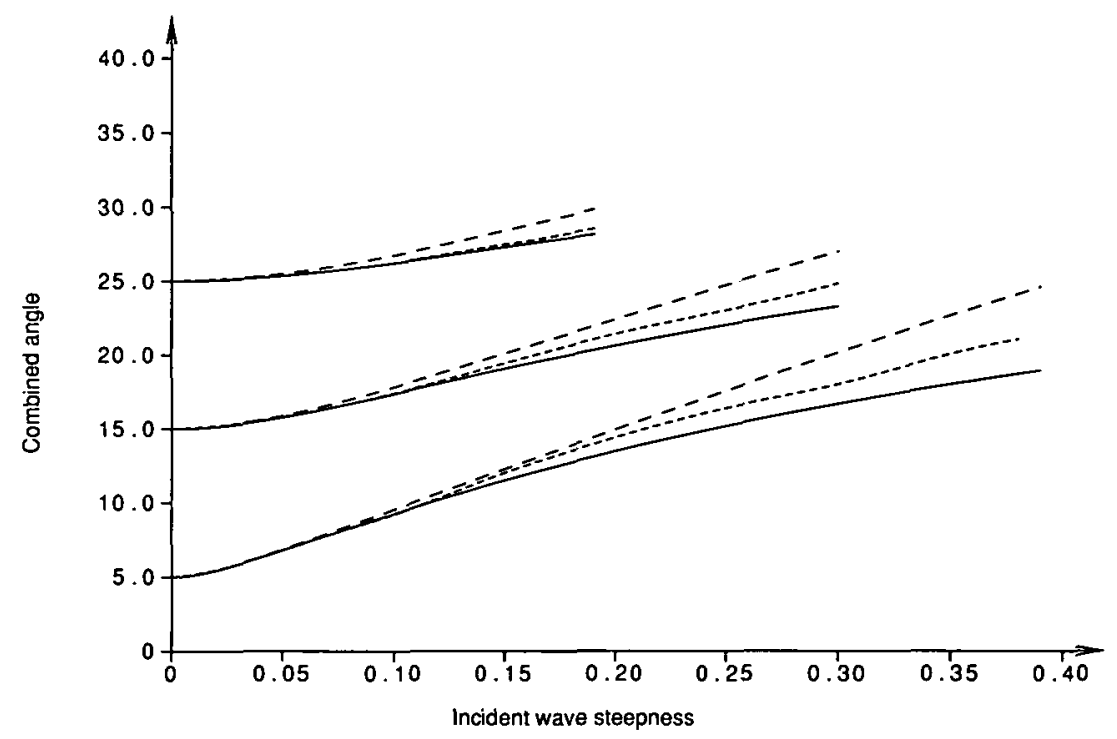

FIGURE 3. Combined angle $j+\alpha$ versus the incident wave steepness $a_{I 1}$ for Mach-reflection. Shown is the present theory $(-)$ for wedge angles $\alpha=5^{\circ}, 15^{\circ}$ and $25^{\circ}$. Shown for comparison are the results of Peregrine [9] (- - -) and Yue and Mei [17] (- - ).

Yue and Mei's wave jump model (their Section 4) is weakly-nonlinear, as in the parabolic assumption, and valid for small wedge angle $\alpha$.

Figure 3 shows our results for the combined angle $j+\alpha$ versus incident wave steepness $a_{l 1} k$ for $\alpha=5^{\circ}, 15^{\circ}$ and $25^{\circ}$ (the relevent wedge angle $\alpha$ is the zero amplitude limit of each curve). These results are compared against both Peregrine's and Yue and Mei's results. Our results compare well except for some deviation at higher wave steepnesses. Figure 4 shows our results for the wave steepness behind the wave jump $a_{1} k$ versus incident wave steepness $a_{I 1} k$ for the same wedge angles $\alpha$ as in Figure 3. The results in both figures are plotted to the maximum steepness for progressive waves (as given by Cokelet [2]). Figure 4 shows that behind the wave jump the wave is steeper than the incident wave. Indeed, even for very small incident waves the waves behind the wave jump are of finite amplitude, with the amplitude being proportional to the wedge angle $\alpha$ (see Section 4.1). As the wedge angle $\alpha$ increases the range of initial wave steepness for which a solution is possible decreases until, beyond $\alpha \approx 30^{\circ}$, no solution is possible.

Also of interest are the ray paths of the wavefield. In the linear theory the ray paths correspond to the characteristics which are parallel to the wavenumber $\mathbf{k}$. However, nonlinearity splits the characteristics into two different directions (this can be seen in Section 4 for small amplitude waves or in Peregrine ([9], Section 5) for a hydraulic analogy). These characteristic directions can be found by considering the equations (which are the one-phase 


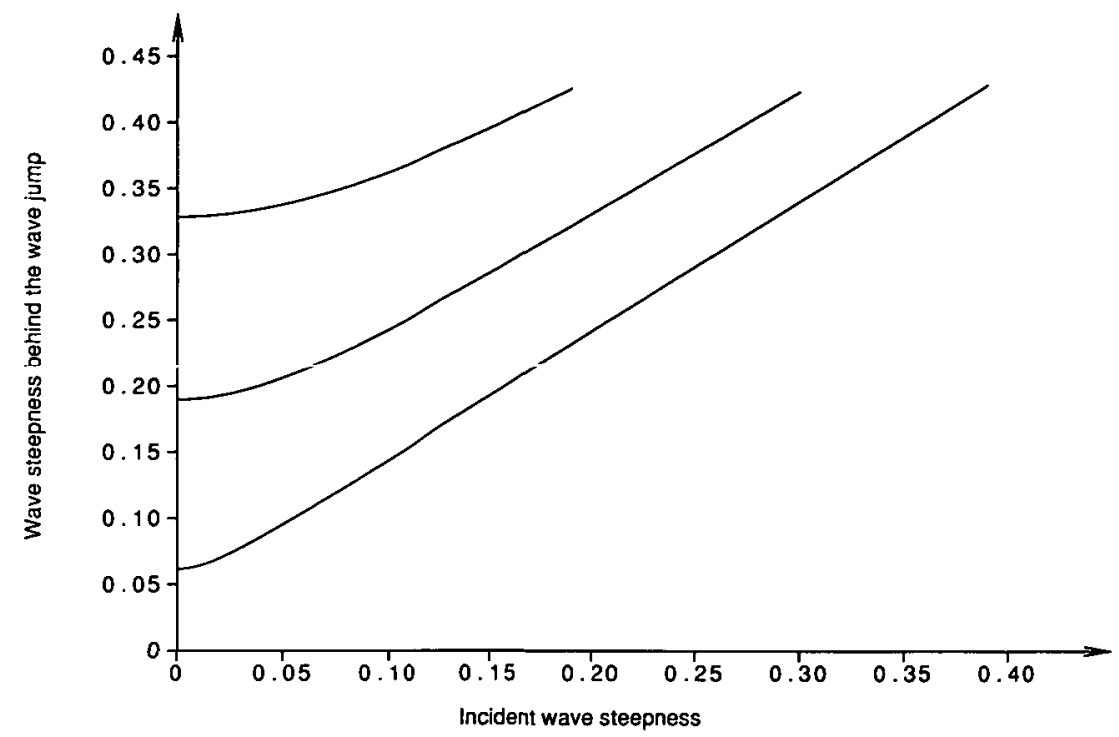

FIGURE 4. The wave steepness behind the wave jump $a_{1} k$ versus the incident wave steepness $a_{I 1}$ for Mach-reflection. Shown is the present theory for wedge angles $\alpha=5^{\circ}, 15^{\circ}$ and $25^{\circ}$. As $\alpha$ increases the curves go from the bottom to the top of the figure.

equivalent of (2.10) and (2.11))

$$
\bar{L}_{a_{i}}=0, \bar{L}_{b_{i}}=0, i=1,2 \text {, }
$$

and

$$
\nabla \times \mathbf{k}=\mathbf{0} \quad \omega=\text { constant } \quad \nabla \cdot \bar{L}_{\mathbf{k}}=0,
$$

which can be written in the form

$$
A \mathbf{v}_{x}+B \mathbf{v}_{y}=0
$$

where $\mathbf{v}^{t}=\left(a_{1}, a_{2}, b_{1}, b_{2}, k_{x}, k_{y}\right)$. (6.1) and (6.2) comprise two differential equations and four algebraic equations and hence if the equations are hyperbolic then there will be two characteristic directions. To write them in the form (6.3) requires that the algebraic equations be differentiated, with respect to $x$ say, so they can be written as a differential equation. Then by solving for the eigenvalues $\lambda_{i}$ of the system $B-\lambda A$ the characteristic directions $d y / d x=\lambda_{i}$ are obtained (zero eigenvalues corresponding to the differentiation of (6.1) can be ignored).

Figure 5 shows lines which are parallel to the wavenumber vector $\mathbf{k}$ which we shall call wavelines: in linear theory these wavelines correspond to the ray paths; in this one-phase theory they are streamlines of the wave motion (but this is not true for multi-phase wavetrains). Presented are results for the case $\alpha=5^{\circ}$ and $j=8.5^{\circ}$. The incident waves propagate head-on to the wedge 


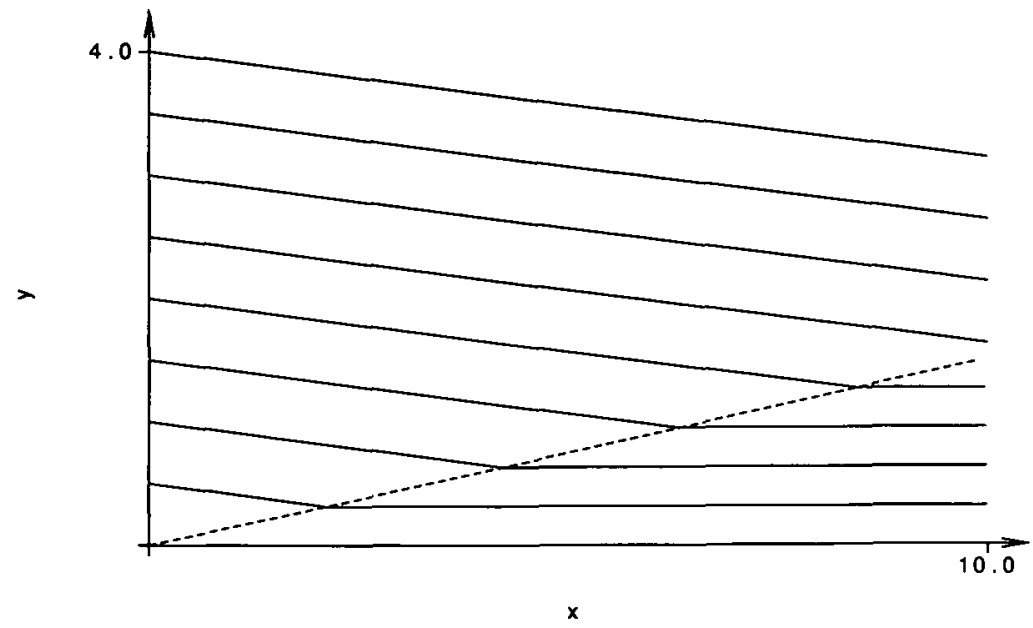

FIGURE 5. The wavelines of the wavefield for wedge angle $\alpha=5^{\circ}$ and jump angle $j=8.5^{\circ}$ for Mach-reflection. The wave jump is drawn along $y=\tan (j) x$ and the $y$-axis is stretched by a factor of 1.5 .

and are refracted through the wave jump to travel parallel to the wedge after the jump. After the wave jump the wavelines are closer together; this reflects the fact that the wedge accumulates the wave energy to some extent and hence the wave steepness is greater after the wave jump than in the incident wavefield. These basic features can also be seen in Figure 6, a contour plot of the wave elevation $\eta$ for the case described above, namely $\alpha=5^{\circ}$ and $j=8.5^{\circ}$. The wave jump can be clearly seen as a line across which a large increase in waveheight occurs. As for the linear case this nonlinear theory does not give any detail of the refraction which occurs around the wave jump.

Figure 7 shows some characteristics of the wavefield as given by (6.3) for this same case of $\alpha=5^{\circ}$ and $j=8.5^{\circ}$. Firstly it is seen again that the ray paths of linear theory have split into two families of nonlinear characteristics. This can be clearly seen in the incident wavefield where the two families of characteristics beginning at the line $x=0$ diverge from each other. Behind the wave jump one family of characteristics emanate from the wedge while the other family come from the characteristics of the incident wavefield transmitted through the wave jump. The wave jump is formed by the intersection of the characteristics corresponding to the solid lines. Qualitatively then, the example described here is similar to the small amplitude one-phase solution described in Section 4.1. 


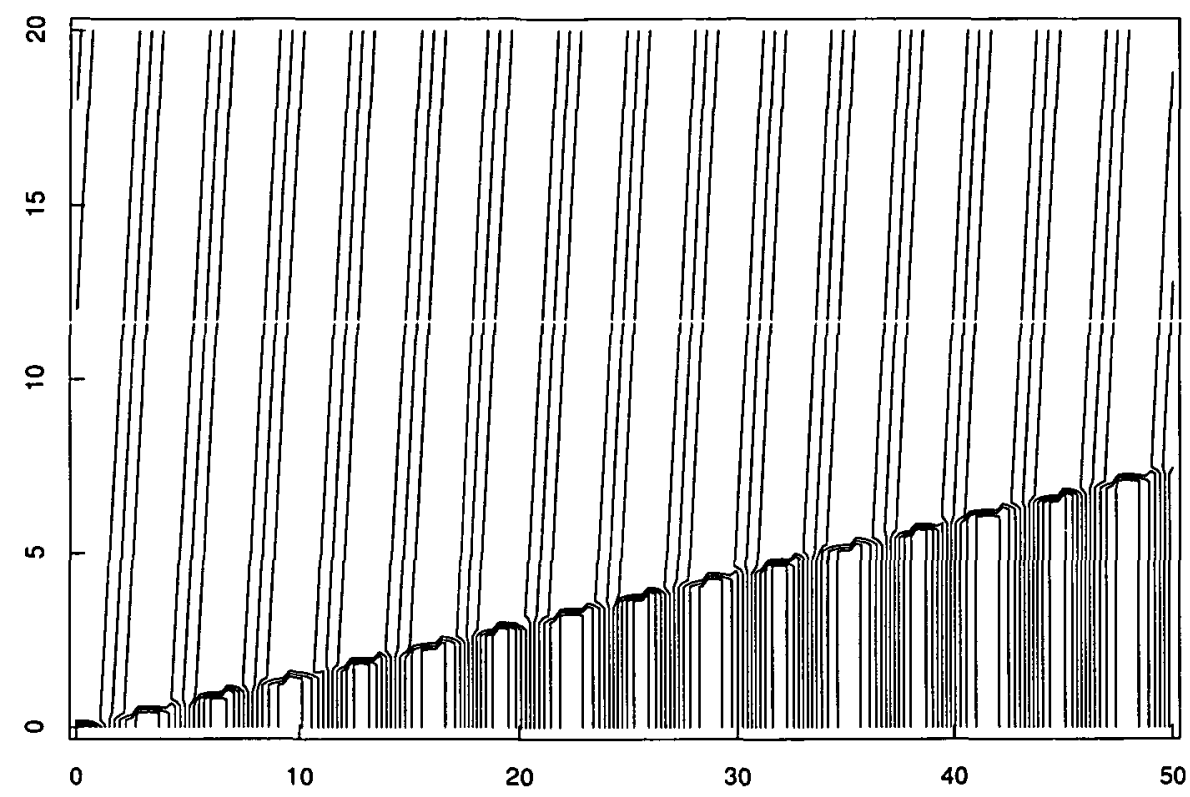

Figure 6. Drawn is a contour plot of the surface elevation $\eta$ for wedge angle $\alpha=5^{\circ}$ and jump angle $j=8.5^{\circ}$ for Mach-reflection. The wedge tip is in the bottom left hand corner of the figure and the $y$-axis has been stretched by a factor of 1.5 .

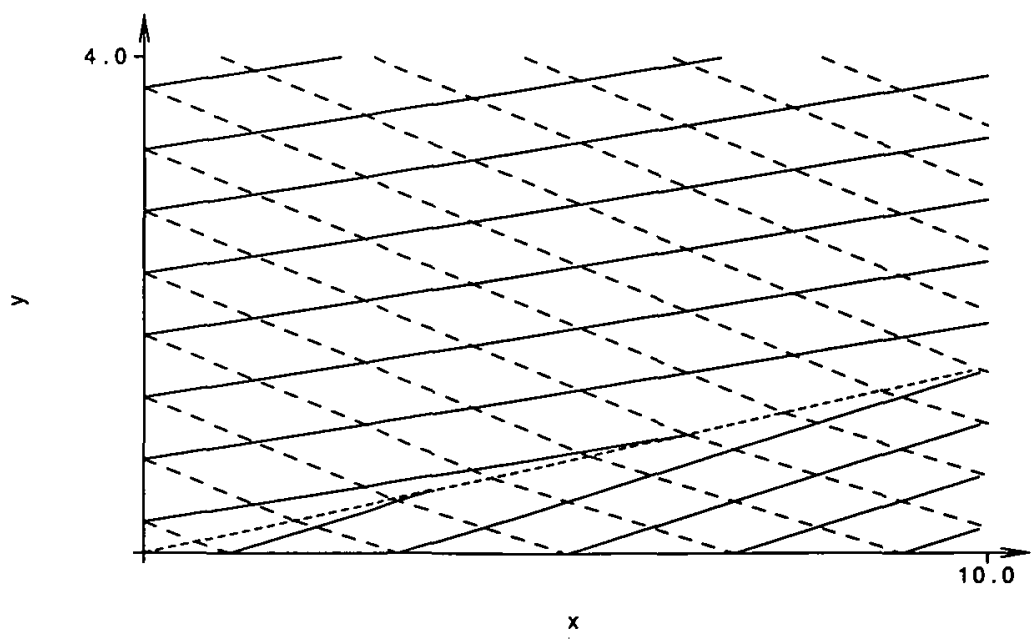

Figure 7. The nonlinear ray paths of the wavefield for wedge angle $\alpha=5^{\circ}$ and jump angle $j=8.5^{\circ}$ for Mach-reflection. The wave jump is drawn along $y=\tan (j) x$ and the $y$-axis has been stretched by a factor of 1.5 . 


\section{Regular and near Mach-reflection}

Here the model which involves reflection from the wedge, and thus possesses both an incident and reflected wave, is considered. Figure 8 shows the numerical results for combined angle $j+\alpha$ versus incident wave steepness $a_{l 1} k$ for wedge angles $\alpha=5^{\circ}, 10^{\circ}$ and $20^{\circ}$ while Figure 9 shows the results for the wave steepness (of one wavetrain) behind the wave jump $a_{1} k$ versus the incident wave steepness $a_{I 1} k$ for the same wedge angles $\alpha$ as in Figure 8. The results show that for small wedge angles there exist two different solutions for each wedge angle $\alpha$ and for a range of incident wave steepnesses.

The solution branch of lower jump angle has a steep long-crested wavefield (as defined in Roberts and Peregrine [13]) behind the wave jump. In this case the incident wavetrain is bent through the wave jump to strike the wedge glancingly. This solution branch is similar to the solutions of Section 6 and is thus called near Mach-reflection (the small amplitude and small wedge angle $\alpha$ limit was discussed in Section 4.3). However, it is different as it involves two distinct wavetrains behind the wave jump.

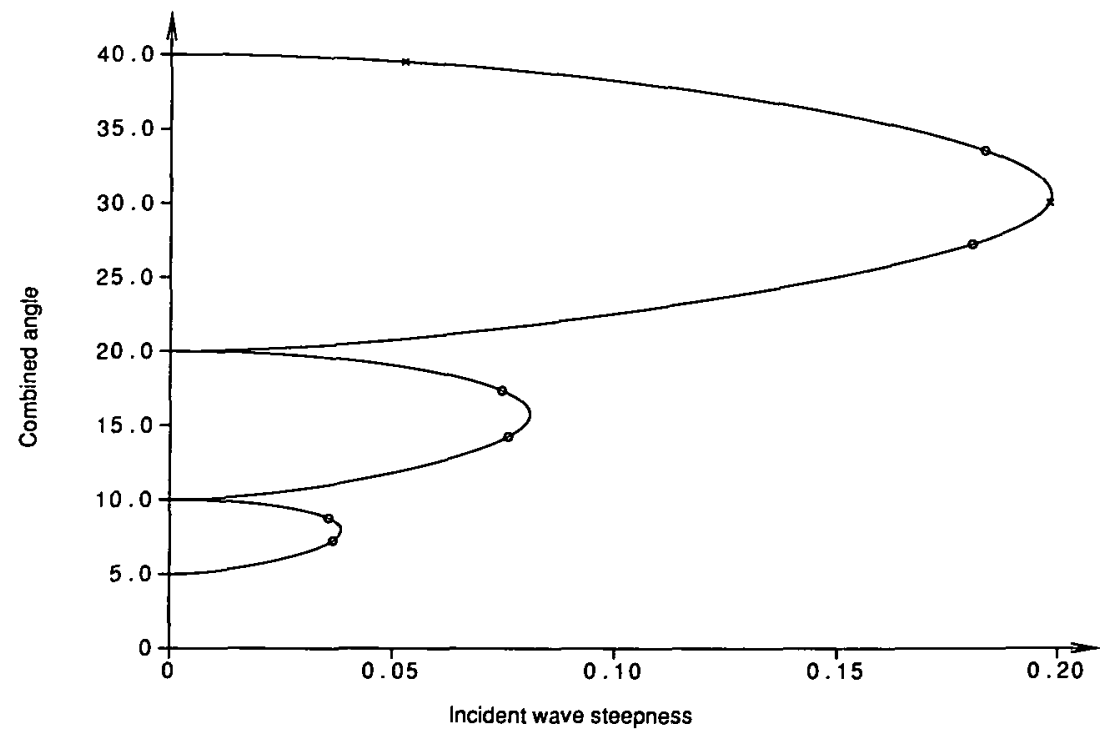

FIGURE 8. Combined angle $j+\alpha$ versus the incident wave steepness $a_{l 1}$ for regular and near Mach-reflection. Shown is the present theory for wedge angles $\alpha=5^{\circ}, \alpha=10^{\circ}$ and $20^{\circ}$. The circles on the curves represent the junctions between the regular and near Mach-reflection regimes while the crosses represent Example $A$ (on the solution branch of higher jump angle) and Example $B$. 


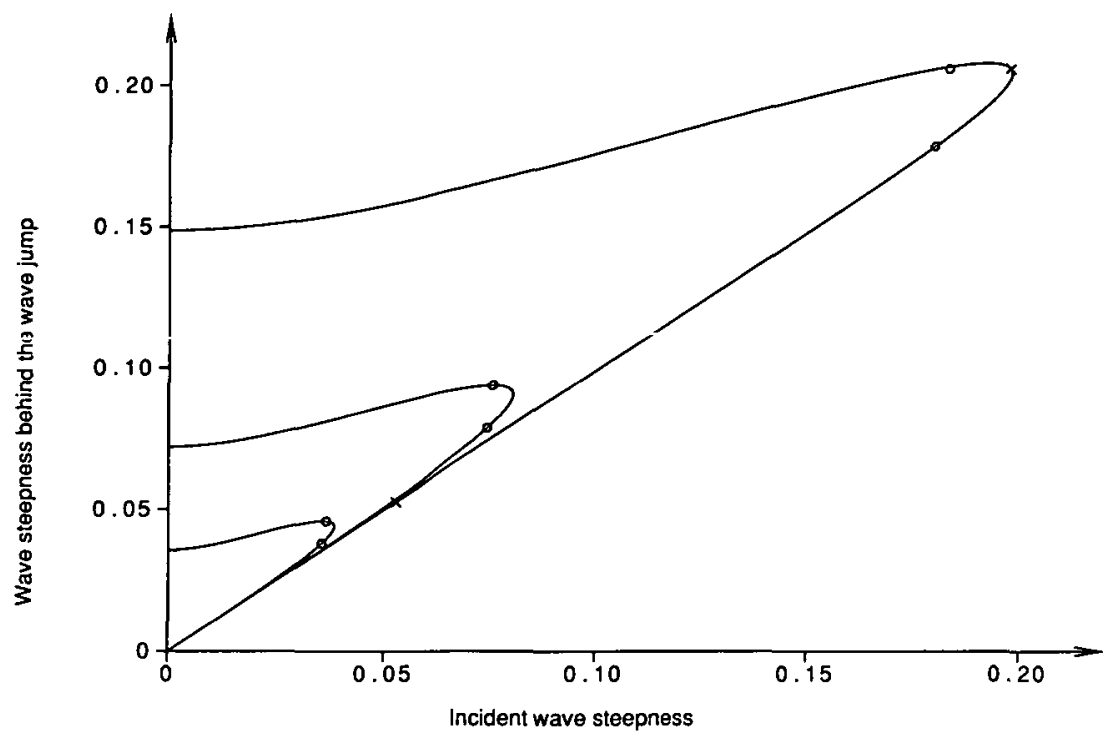

FIGURE 9. The wave steepness behind the wave jump $a_{1} k$ versus the incident wave steepness $a_{I 1}$ for regular and near Mach-reflection. Shown is the present theory for wedge angles $\alpha=5^{\circ}$, $\alpha=10^{\circ}$ and $20^{\circ}$ (the wave steepness of the near Mach-reflection solution branch increases as $\alpha$ increases). The circles on the curves represent the junctions between the regular and near Mach-reflection regimes while the crosses represent Example A (on the solution branch of lower wave steepness) and Example B.

The solution branch of higher jump angle is qualitatively similar to the regular reflection of linear waves (the small amplitude and small wedge angle limit was discussed in Section 4.2). The incident wavetrain is only slightly bent through the wave jump and the wave steepness is little changed. The jump angle is approximately the same as the wedge angle, but decreases with increasing incident wave steepness.

The theory also provides nonlinear solutions for blunt wedges. Figure 10 shows the results for the combined angle $j+\alpha$ versus the incident wave steepness $a_{I 1} k$ for $\alpha=30^{\circ}, 50^{\circ}$ and $70^{\circ}$. The curves are plotted until the maximum steepness of the corresponding short-crested wavefield behind the wavejump is reached (using estimates from Roberts [12]). Here only the solution branch of regular reflection exists because solutions corresponding to near Mach-reflection would have wave steepness behind the wave jump very much greater than the maximum steepness of water waves.

Figure 11 shows the Mach-reflection, regular reflection and near Machreflection solution branches of the combined angle $j+\alpha$ versus the incident wave steepness $a_{I 1} k$ for $\alpha=15^{\circ}$ which is a typical picture for thin wedges. This shows that at low incident wave steepness there exists three possible solutions. In practice it would be expected that the regular reflec- 


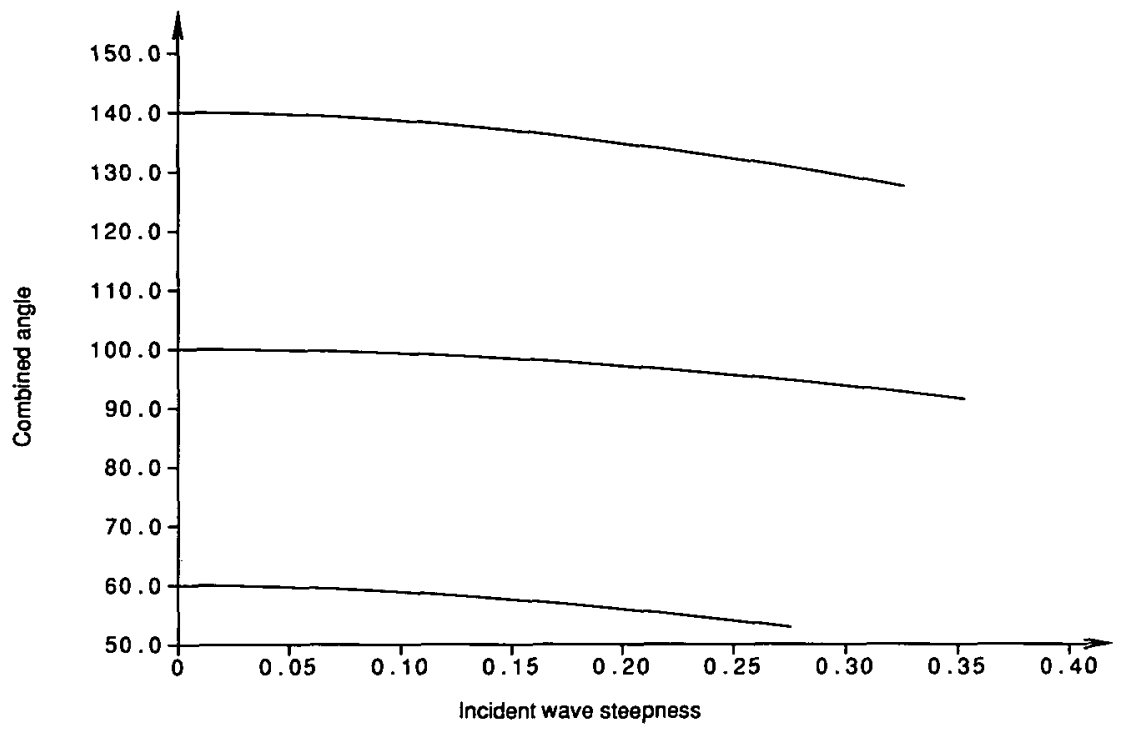

FIGURE 10. Combined angle $j+\alpha$ versus the incident wave steepness $a_{l 1}$ for regular reflection. Shown is the present theory for wedge angles $\alpha=30^{\circ}, 50^{\circ}$ an $70^{\circ}$.

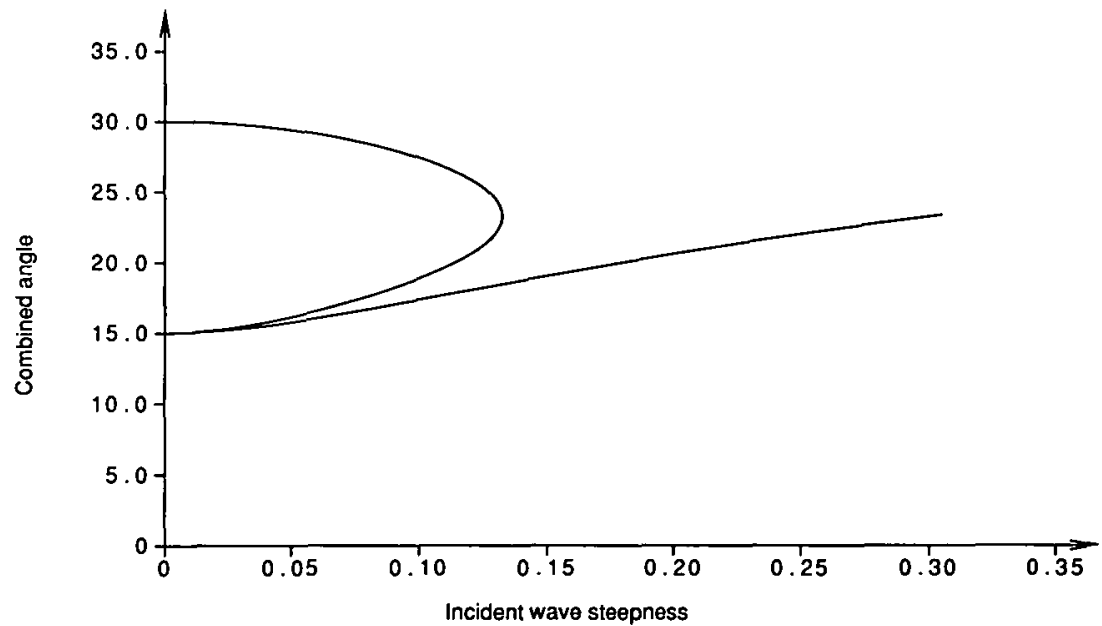

FIGURE 11. Combined angle $j+\alpha$ versus the incident wave steepness $a_{I 1}$ for Mach-reflection (the bottom curve), regular and near-Mach reflection. Shown is the present theory for wedge angle $\alpha=15^{\circ}$.

tion branch would occur for low wave steepnesses. As the wave steepness increases the physically realised solution would transit to the Mach-reflection solution. This is consistent with the solutions of Kirby and Dalrymple [4] for their wedge shaped depression. Their solutions shows that at a small incident wave steepness the nonlinear solution is similar to the linear solution (i.e. the 
solution is on the regular reflection branch) and for an increased incident wave steepness the solution corresponds to Mach-reflection.

However, the possibility of hysteresis exists. For instance, if the wavefield is on the Mach-reflection branch and the wave steepness is decreased to zero, then the wavefield could remain in the form of Mach-reflection. However, as the wave steepness approaches zero, the Mach-reflection solution probably becomes less stable and a small perturbation would cause the wavefield to transit back to the regular reflection branch. In this scenario we would expect the near Mach-reflection branch to be unstable, but this is as yet unknown.

So at small incident wave steepnesses (corresponding to about $K<0.5$ in deep water for Yue and Mei's [18] analysis) it is possible that numerical solutions of the nonlinear Schrödinger equation could correspond to any of the three solution branches (for incident wave steepnesses beyond about $K=$ 0.5 only Mach-reflection is possible). Yue and Mei interpret their solutions at $K=0$ and $K=0.5$ as Mach-reflection (see their Figure 8) which indicates that hysterisis occurs. However, the linear solution (when $K=0$ ) is just the result of the superposition of the incident and reflected wavetrains hence it corresponds to regular reflection. So at small $K$ the solution is hard to characterise due to the diffractive and refractive effects causing the position of the wave jump to be very indistinct. The farfield solution (where the existence of a Mach-stem would be more noticeable) would help resolve this difficulty.

Another point of interest is the refraction of the incident wavetrain through the wave jump. Figure 12 shows the results for $\Theta$ versus the incident wave steepness $a_{11} k$ for $\alpha=5^{\circ}, \alpha=10^{\circ}$ and $20^{\circ}$ where $\Theta$ represents the angle which the incident wavetrain behind the wave jump makes with the wedge. For regular reflection the incident wavetrain is slightly bent away from the wedge as it goes through the wave jump, this effect increases as the incident wave steepness increases. For near Mach-reflection the incident wavetrain is bent through the wave jump to travel nearly (but not quite) parallel to the wedge; this bending lessens (the wavetrain is at a greater angle to the wedge) as the incident wave steepness increases.

To examine the transition between regular and near Mach-reflection two examples (marked with crosses on the appropriate curves in Figure 8 and 9) for wedge angle $\alpha=20^{\circ}$ are considered. Example A with jump angle $j=19.5^{\circ}$ is an example of regular reflection while, Example B with a jump angle $j=10^{\circ}$ is an example of solutions in the transition region between regular and near Mach-reflection.

The characteristics for the short-crested wavefield behind the wave jump can be found by considering (2.10) and (2.11). Using the symmetry between the incident and reflected waves the differential and algebraic equations cor- 


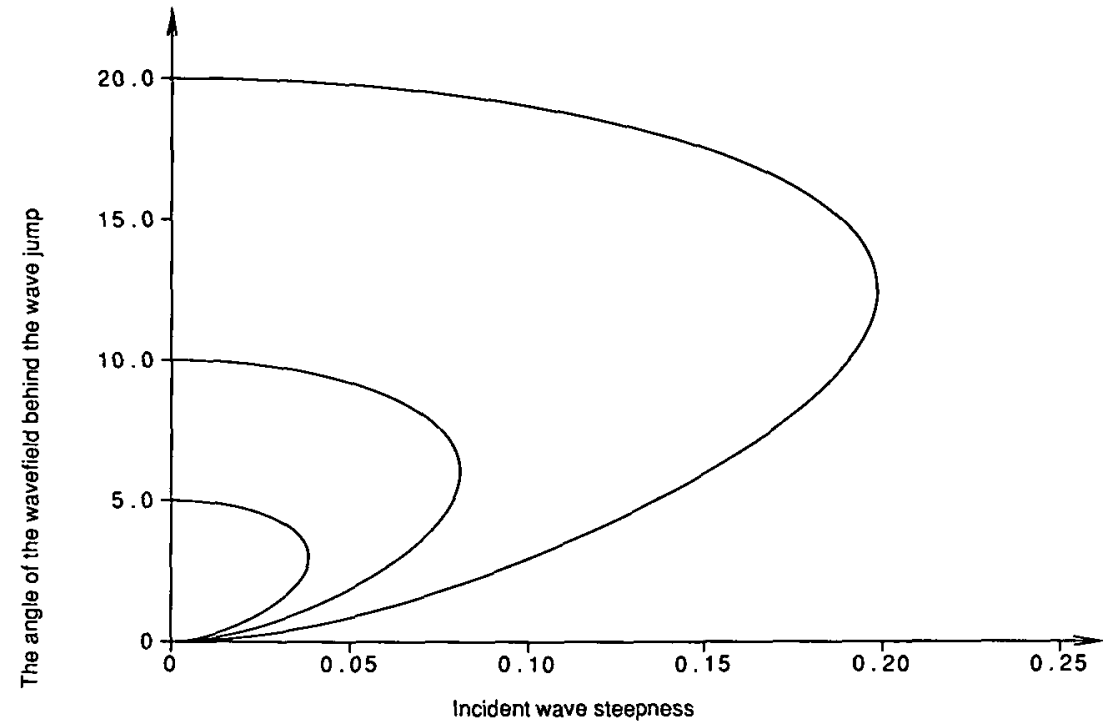

FIGURE 12. The angle between the wedge and the incident wavetrain behind the wave jump $\theta$ versus the incident wave steepness $a_{I 1}$ for regular and near Mach-reflection. Shown is the present theory for wedge angles $\alpha=5^{\circ}$ (the inner curve), $\alpha=10^{\circ}$ and $20^{\circ}$ (the outer curve).

responding to the incident wavefield can be written

$$
\bar{L}_{a_{i}}=0 \quad \bar{L}_{b_{i}}=0 \quad i=1,3,5,6,
$$

and

$$
\nabla \times \mathbf{k}=\mathbf{0} \quad \omega=\text { constant } \quad \nabla \cdot \bar{L}_{\mathbf{k}}=0,
$$

and hence may be written as

$$
A \mathbf{v}_{x}+B \mathbf{v}_{y}=0
$$

where $\mathbf{v}^{1}=\left(a_{1}, a_{3}, a_{5}, a_{6}, b_{1}, b_{3}, b_{5}, b_{6}, k_{x}, k_{y}\right)$. (7.1) and (7.2) comprise two differential equations and eight algebraic equations. Thus if the equations are hyperbolic then there will be two characteristic directions for the incident wavefield. Hence by solving for the eigenvalues $\lambda_{i}$ of the system $B-\lambda A$ the characteristic directions $d y / d x=\lambda_{i}$ are obtained (the zero eigenvalues can be ignored as in Section 6). Because of the symmetry of the wavefield the ray paths corresponding to the reflected wavetrain will have characteristic directions $d y / d x=-\lambda_{i}$.

Figure 13 shows the wavelines for Example $A$ which is an example of regular reflection. All the figures drawn for Examples $\mathrm{A}$ and $\mathrm{B}$ have the $y$ direction scaled slightly, hence the wedge appears broader than in reality. The incident waves are only slightly refracted through the wave jump and a short-crested wavefield is set up between the wave jump and the wedge. Figure 14 shows a contour plot of the wave elevation $\eta$ for this example. The 


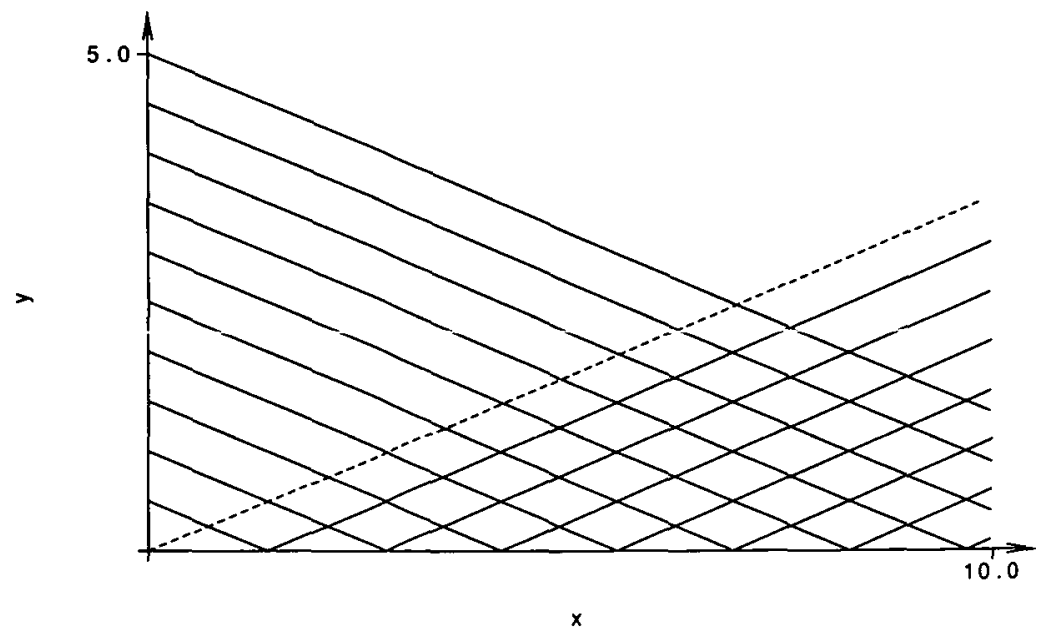

Figure 13. The wavelines of the wavefield for Example A which has wedge angle $\alpha=20^{\circ}$ and jump angle $j=19.5^{\circ}$. The wave jump is drawn along $y=\tan (j) x$ and the $y$-axis is stretched by a factor of 1.2 .

wave jump which divides the progressive wavefield from the short-crested wavefield next to the wedge is clearly visible. The short-crested wavefield consists of short steep waves with flat troughs (as $\alpha=20^{\circ}$ in this example, the wave crests are already starting to lengthen in the $y$-direction). Figure 15 (a) and 15(b) show the two families of characteristics for the incident and reflected wavefields respectively. Again the two families of characteristics corresponding to the incident wavetrain show the typical nonlinear splitting and are only slightly bent through the wave jump. For the reflected wavetrain both families of characteristics emanate at the wedge. The family of characteristics shown by the solid lines propagate to the wave jump, while the family shown by the dashed lines do not reach the wave jump. Hence some of the family of characteristics shown by the dashed lines also emanate at the wave jump. Example A then, is qualitatively similar to the two-phase smallamplitude solution described in Section 4.2 and is just a slight modification of the linear ray theory.

Figure 16 shows the wavelines for Example B. This example is in the transition region between regular and near Mach-reflection. The incident waves are refracted to a significant degree after the wave jump and the wavefield behind the wave jump is becoming long-crested. Figure 17, a contour plot of the wave elevation $\eta$, shows that the waves next to the wedge are now very long in the $y$-direction with only a little transverse structure evident in the wavefield. Figure 18(a) and 18(b) show the two families of characteristics for the incident and reflected wavefields respectively. Since the incident wave 


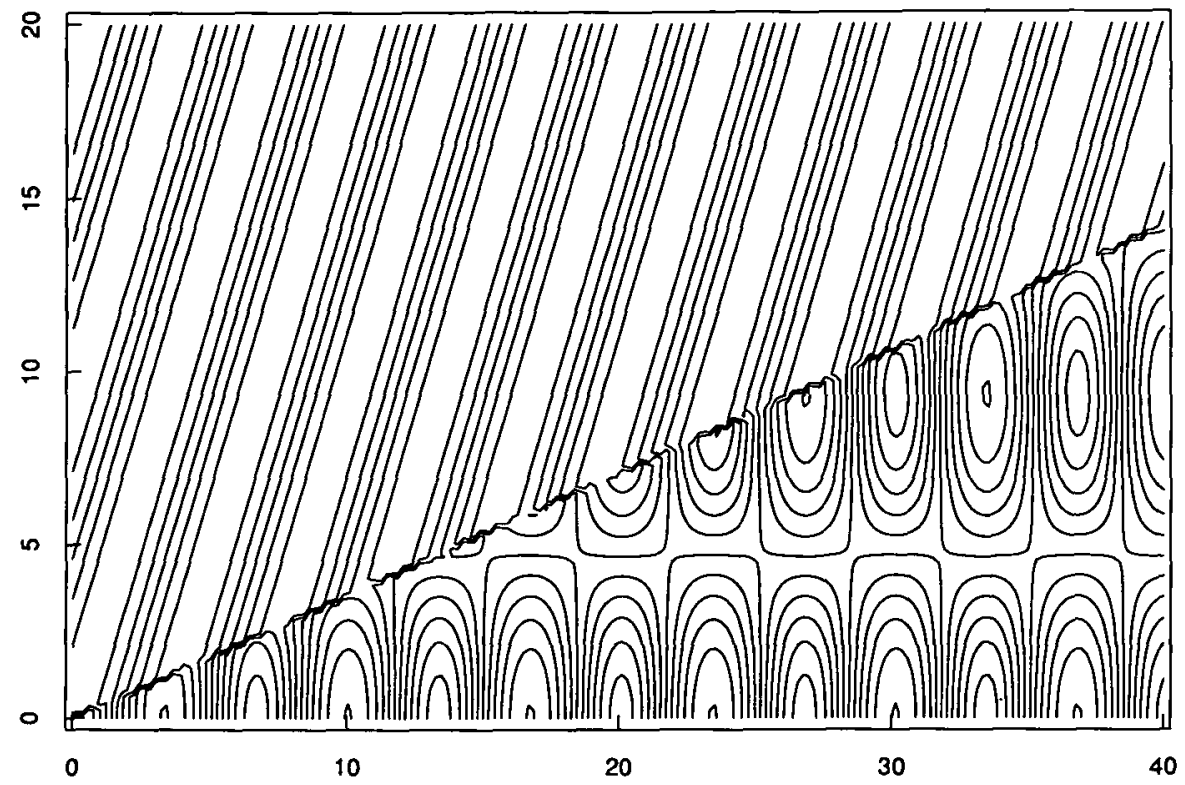

FIGURE 14. Drawn is a contour plot of the surface elevation $\eta$ for Example $A$ which has wedge angle $\alpha=20^{\circ}$ and jump angle $j=19.5^{\circ}$. The wedge tip is in the bottom left hand corner of the figure and the $y$-axis is stretched by a factor of 1.3 .

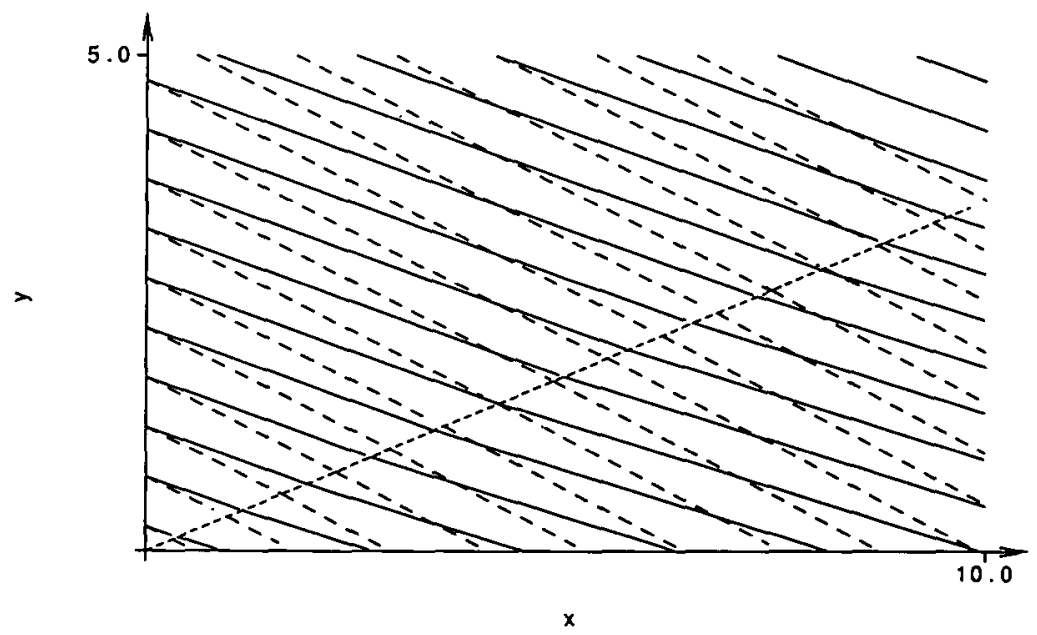

FIGURE 15(a). The nonlinear ray paths of the incident wavefield for Example $A$ which has wedge angle $\alpha=20^{\circ}$ and jump angle $j=19.5^{\circ}$. The wave jump is drawn along $y=\tan (j) x$ and the $y$-axis is stretched by a factor of 1.2 .

steepness is larger here, the two families of characteristics corresponding to the incident wavetrain are now split even wider. Behind the wave jump the family of characteristics corresponding to the solid lines, associated with the 


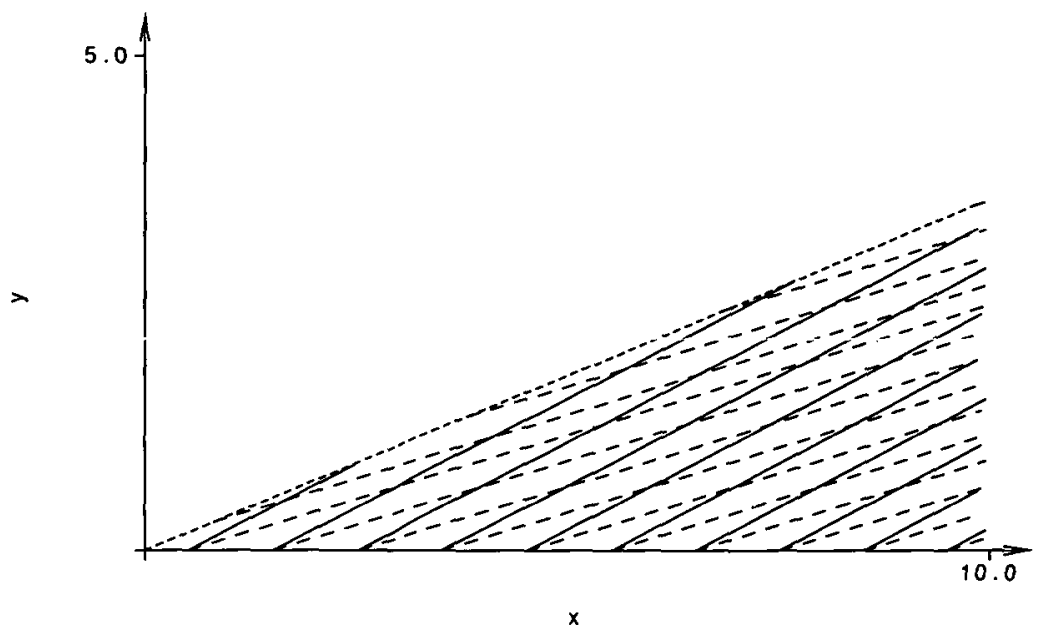

FIGURE 15(b). The nonlinear ray paths of the reflected wavefield for Example A which has wedge angle $\alpha=20^{\circ}$ and jump angle $j=19.5^{\circ}$. The wave jump is drawn along $y=\tan (j) x$ and the $y$-axis is stretched by a factor of 1.2 .

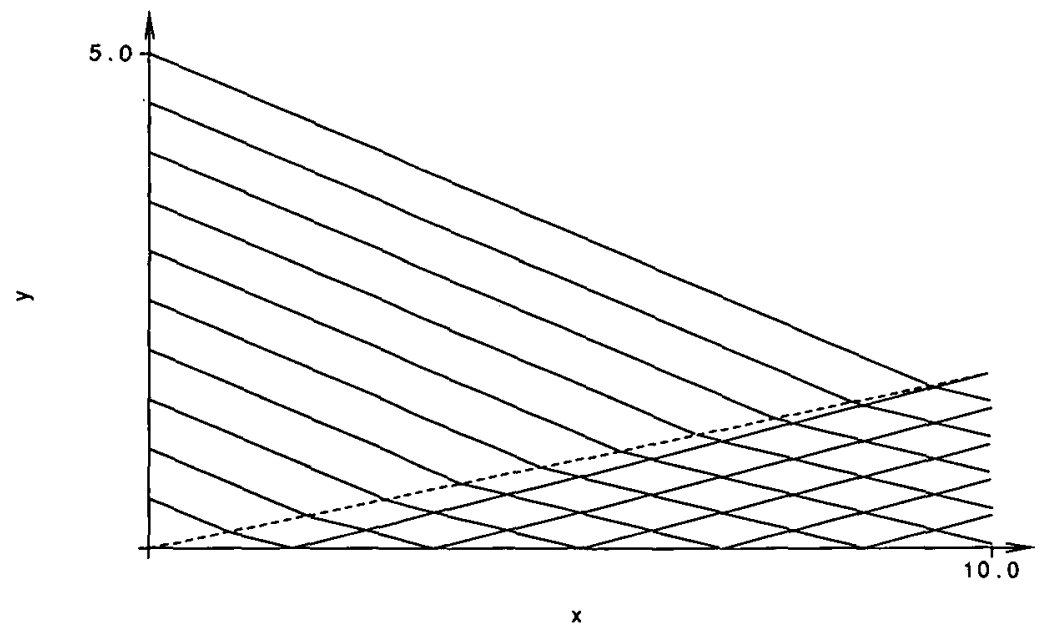

FigURE 16. The wavelines of the wavefield for Example B which has wedge angle $\alpha=20^{\circ}$ and jump angle $j=10^{\circ}$. The wave jump is drawn along $y=\tan (j) x$ and the $y$-axis is stretched by a factor of 1.2 .

incident wavefield do not travel towards the wedge, hence some characteristics of this family emanate at the wedge while the other emanates at the wave jump. This picture of the characteristics then, is qualitatively different from Example A.

The point when the picture of the characteristics changes is when one family of characteristics travels parallel to the wedge. The circle on the solution 


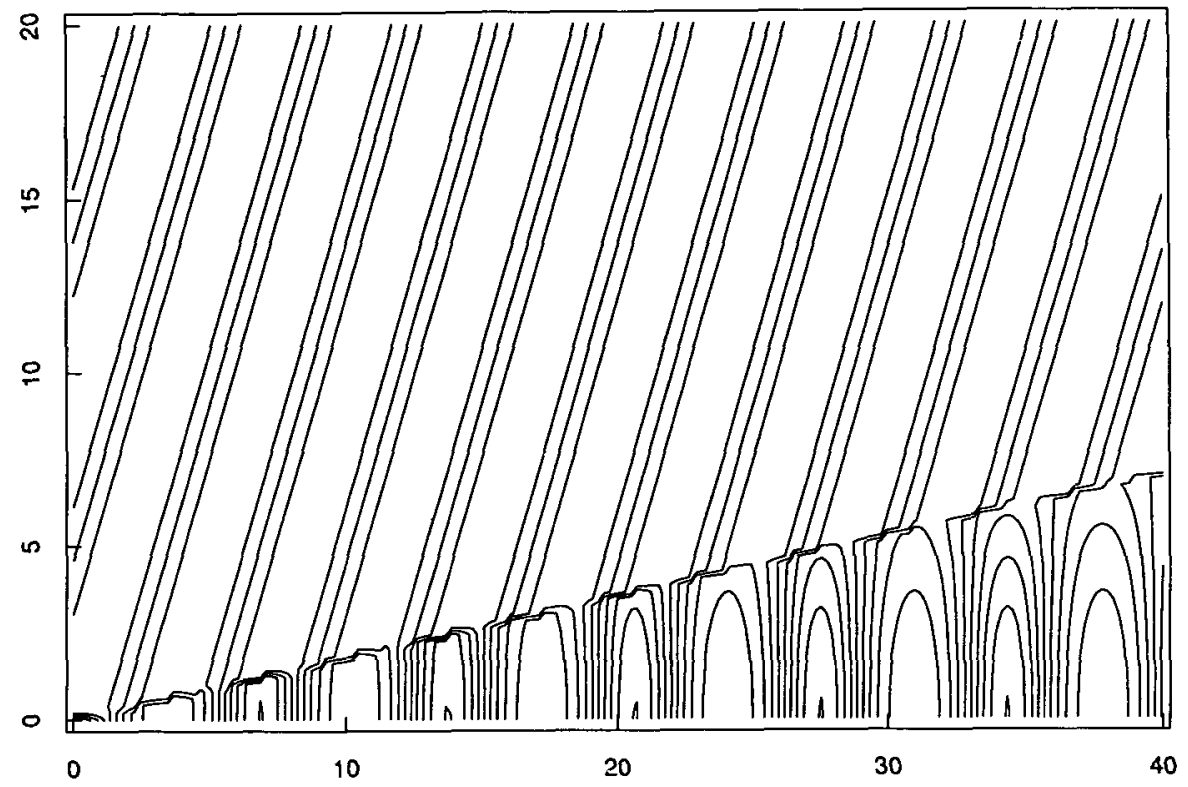

FiguRE 17. Drawn is a contour plot of the surface elevation $\eta$ for Example B which has wedge angle $\alpha=20^{\circ}$ and jump angle $j=10^{\circ}$. The wedge tip is in the bottom left hand corner of the figure and the $y$-axis is stretched by a factor of 1.3 .

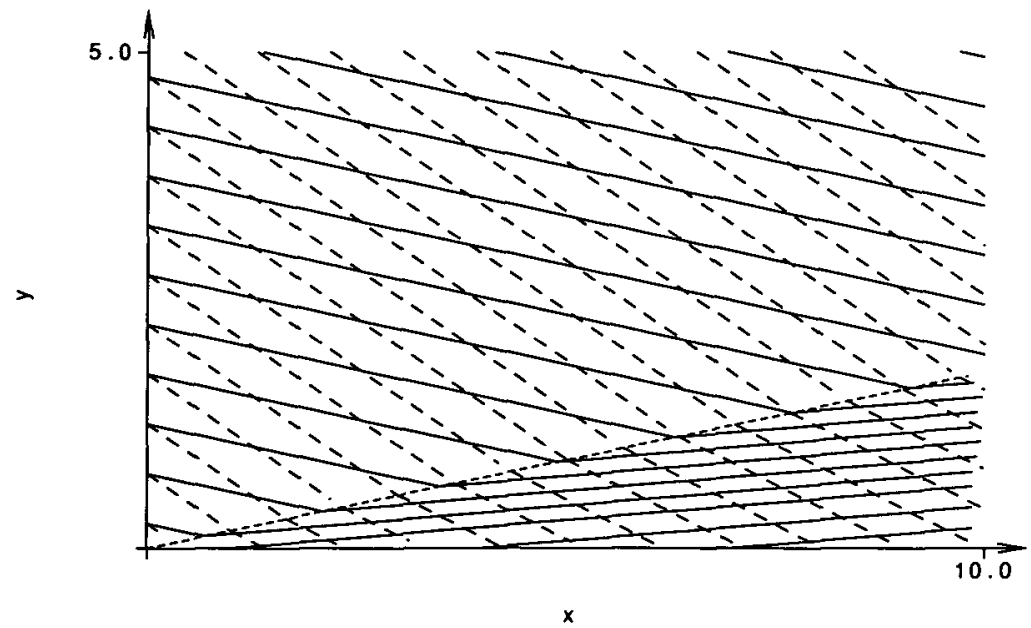

FIGURE 18(a). The nonlinear ray paths of the incident wavefield for Example B which has wedge angle $\alpha=20^{\circ}$ and jump angle $j=10^{\circ}$. The wave jump is drawn along $y=\tan (j) x$ and the $y$-axis is stretched by a factor of 1.2 .

branch of higher jump angle of the curves in Figure 8 and on the solution branch of lower wave steepness of the curves in Figure 9 shows this junction between regular reflection (Example $\mathrm{A}$ ) and solutions in the transition 


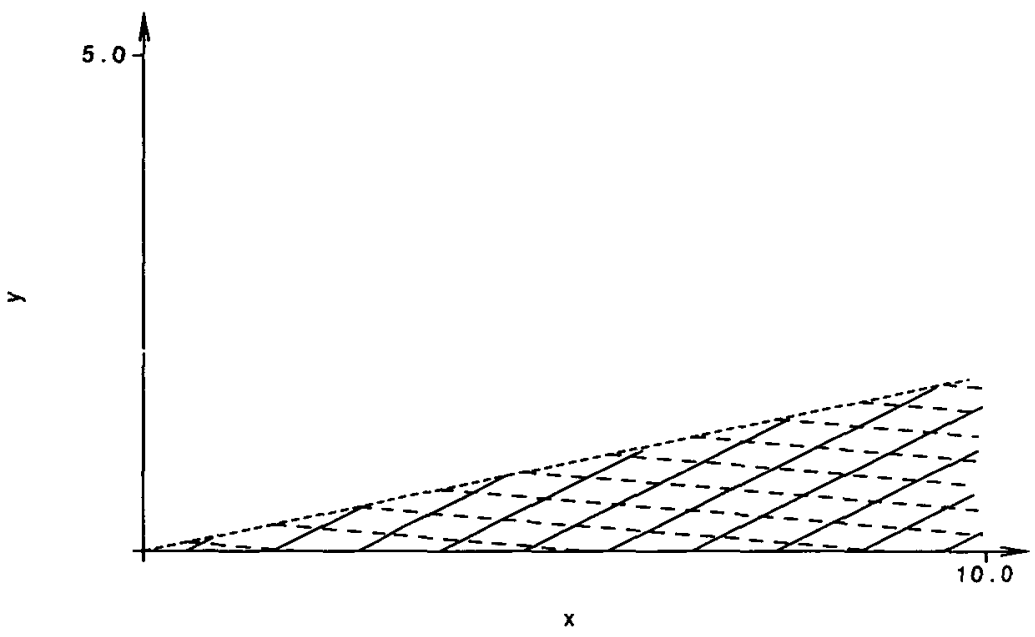

FigURE $18(\mathrm{~b})$. The nonlinear ray paths of the reflected wavefield for Example B which has wedge angle $\alpha=20^{\circ}$ and jump angle $j=10^{\circ}$. The wave jump is drawn along $y=\tan (j) x$ and the $y$-axis is stretched by a factor of 1.2 .

region (Example B). The transition to near Mach-reflection is complete when behind the wavejump, one family of characteristics associated with the incident wavetrain emanate solely at the wedge while the other family emanate from the wave jump (this is the picture of the characteristics for small amplitude near Mach-reflection, see Section 4.3). This point is marked on the solution branch of lower jump angle of the curves in Figure 8 and on the solution branch of higher wave steepness of the curves in Figure 9 by a circle also. In summary, because of the qualitative differences between the pictures of the characteristics the solution curves can be divided into three regions: (a) regular reflection, (b) near Mach-reflection and (c) a transition region between (a) and (b).

The force exerted by the waves upon the wedge is of interest since the wedge can represent the bow of a ship or a breakwater of finite length. Here the depth-integrated force per unit length on the wedge is considered. This has been examined in Marchant and Roberts [6] for short-crested waves striking a breakwater of infinite length in water of finite-depth via a high-order perturbation series. Here the same definitions are used (see their Section 6) and hence a second-order nondimensional depth-integrated force per unit length is calculated. Figure 19 shows the results for incident wave steepness $a_{I 1} k$ versus the maximum force per unit length for wedge angles $\alpha=5^{\circ}, \alpha=10^{\circ}$ and $\alpha=20^{\circ}$. The results correspond closely to that given by second-order perturbation theory and show that for a given incident wavetrain a larger maximum force will result if the wave displays near Mach-reflection rather 


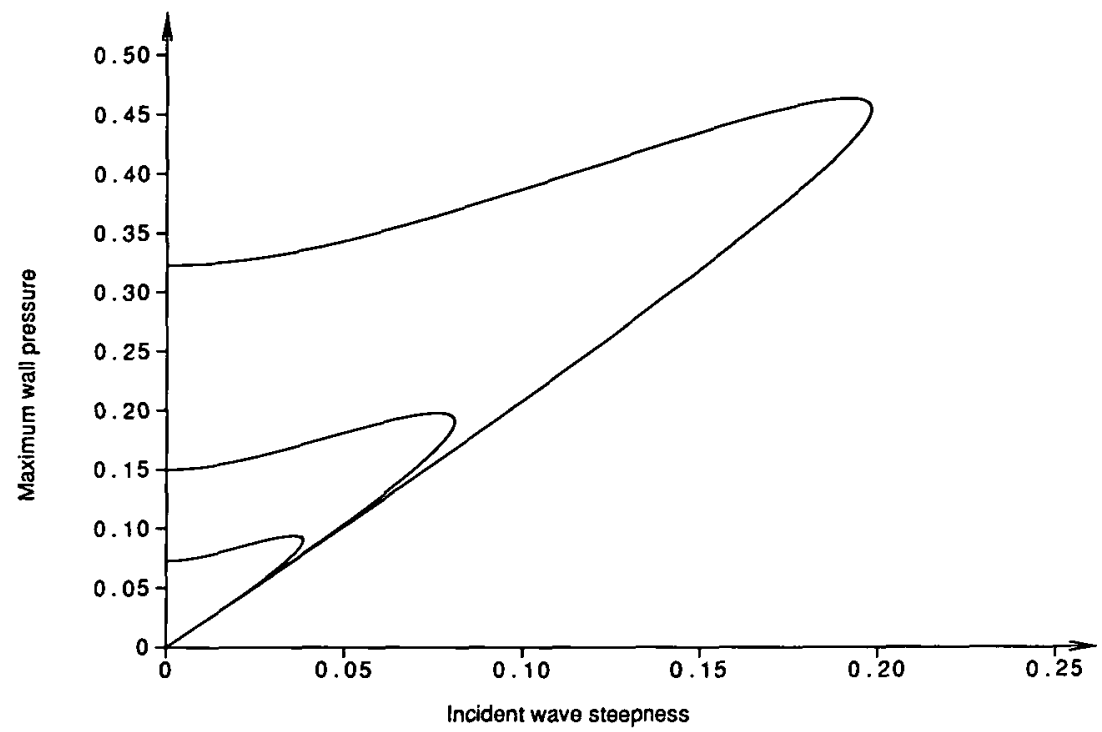

FIGURE 19. The maximum force per unit length versus the incident wave steepness $a_{l 1}$ for regular and near Mach-reflection. Shown is the present theory for wedge angles $\alpha=5^{\circ}$ (the inner curve), $\alpha=10^{\circ}$ and $20^{\circ}$ (the outer curve).

than regular reflection since the wave steepness next to the wedge is greater for the near Mach-reflection case.

In addition, solutions for waves approaching the wedge at an angle, other than head on, can be found (for example, where the bow of the ship heads at an angle into the waves). Because of the hyperbolic nature of the equations, the wavefield on one side of the wedge is not affected by the wavefield on the other side. The characteristics corresponding to the reflected wavetrain exist only behind the wave jump and do not interact with the incident wave ahead of the wedge nor do they interact with the characteristics on the other side of the wedge. Hence the solutions for two different wedge angles can be patched together to give the solution for waves approaching the wedge at an angle.

\section{Appendix}

The averaged Lagrangian was calculated with the aid of REDUCE, an algebraic manipulation package. The averaged Lagrangian for the incident 
progressive wavefield is

$$
\begin{aligned}
\bar{L}_{I}= & -(1 / 4) w k_{I}^{2} a_{I 1}^{3} b_{I 1}-2 w k_{I} a_{I 1}^{2} b_{I 3}-w k_{I} a_{I 1} a_{I 3} b_{I 1}-4 w a_{I 3} b_{I 3} \\
& +k_{I}^{3} a_{I 1}^{2} b_{I 1}^{2}+4 k_{I}^{2} a_{I 1} b_{I 1} b_{I 3}+2 k_{I} b_{I 3}^{2}+a_{I 3}^{2}-2 w a_{I 1} b_{I 1} \\
& +k_{I} b_{I 1}^{2}+a_{I 1}^{2} .
\end{aligned}
$$

The variations of $\bar{L}_{1}$ with the coefficients of the velocity potential $\phi$ are

$$
\begin{aligned}
\bar{L}_{b_{I I}}= & -(1 / 4) w k_{I}^{2} a_{I 1}^{3}-w k_{I} a_{I 1} a_{I 3}-2 w a_{I 1}+2 k_{I}^{3} a_{I 1}^{2} b_{I 1} \\
& +4 k_{I}^{2} a_{I 1} b_{I 3}+2 k_{I} b_{I 1}, \\
\bar{L}_{b_{I 3}}= & -2 w k_{I} a_{I 1}^{2}-4 w a_{I 3}+4 k_{I}^{2} a_{I 1} b_{I 1}+4 k_{I} b_{I 3} .
\end{aligned}
$$

The variations of $\bar{L}_{I}$ with the coefficients of the free surface shape $\eta$ are

$$
\begin{aligned}
\bar{L}_{a_{I 1}=} & -(3 / 4) w k_{I}^{2} a_{I 1}^{2} b_{I 1}-4 w k_{I} a_{I 1} b_{I 3}-w k_{I} a_{I 3} b_{I 1}-2 w b_{I 1} \\
& +2 k_{I}^{3} a_{I 1} b_{I 1}^{2}+4 k_{I}^{2} b_{I 1} b_{I 3}+2 a_{I 1}, \\
\bar{L}_{a_{I 3}=} & -w k_{I} a_{I 1} b_{I 1}-4 w b_{I 3}+2 a_{I 3} .
\end{aligned}
$$

The variations of $\bar{L}_{1}$ with the wavenumber involve

$$
\begin{aligned}
\bar{L}_{k_{I}}= & -(1 / 2) w k_{I} a_{I 1}^{3} b_{I 1}-2 w a_{I 1}^{2} b_{I 3}-w a_{I 1} a_{I 3} b_{I 1}+3 k_{I}^{2} a_{I 1}^{2} b_{I 1}^{2} \\
& +8 k_{I} a_{I 1} b_{I 1} b_{I 3}+b_{I 1}^{2}+2 b_{I 3}^{2},
\end{aligned}
$$

hence

$$
\bar{L}_{k_{l x}}=\bar{L}_{k_{I}} k_{I x} / k_{I}, \quad \bar{L}_{k_{l y}}=\bar{L}_{k_{I}} k_{l y} / k_{I} .
$$

The averaged Lagrangian for the short-crested wavefield is

$$
\begin{aligned}
\bar{L}= & -2 k w a_{1}^{2} b_{3}-k w a_{1} a_{3} b_{1}-k w a_{1} a_{5} b_{1}-k w a_{1} a_{6} b_{1} \\
& +3 k a_{1}^{2} b_{1}^{2} k_{x}^{2}+k a_{1}^{2} b_{1}^{2} k_{y}^{2}+2 k a_{1} b_{1} k_{x} b_{5}+2 k b_{3}^{2} \\
& +k b_{1}^{2}-(3 / 4) w a_{1}^{3} b_{1} k^{2}-2 w a_{1}^{2} k_{x} b_{5}-4 w a_{3} b_{3}-2 w a_{5} b_{5} \\
& +4 a_{1} b_{1} b_{3} k^{2}+2 a_{1} b_{1} k_{x}^{2} b_{5}+a_{3}^{2}+(1 / 2) a_{5}^{2}-a_{5} b_{1}^{2} k_{y}^{2} \\
& +(1 / 2) a_{6}^{2}+a_{6} b_{1}^{2} k_{x}^{2}+(1 / 2) k_{x} b_{5}^{2}+k_{y} b_{6}^{2} \\
& -2 w a_{1} b_{1}+a_{1}^{2} .
\end{aligned}
$$


The variations of $\bar{L}$ with the coefficients of the velocity potential $\phi$ are

$$
\begin{aligned}
\bar{L}_{b_{1}}= & -k w a_{1} a_{3}-k w a_{1} a_{5}-k w a_{1} a_{6}+6 k a_{1}^{2} b_{1} k_{x}^{2}+2 k a_{1}^{2} b_{1} k_{y}^{2} \\
& +2 k a_{1} k_{x} b_{5}+2 k b_{1}-(3 / 4) w a_{1}{ }^{3} k^{2}-2 w a_{1}+4 a_{1} b_{3} k^{2} \\
& +2 a_{1} k_{x}^{2} b_{5}-2 a_{5} b_{1} k_{y}^{2}+2 a_{6} b_{1} k_{x}^{2}, \\
\bar{L}_{b_{3}}= & -2 k w a_{1}^{2}+4 k b_{3}-4 w a_{3}+4 a_{1} b_{1} k^{2} \\
\bar{L}_{b_{5}}= & 2 k a_{1} b_{1} k_{x}-2 w a_{1}^{2} k_{x}-2 w a_{5}+2 a_{1} b_{1} k_{x}^{2}+2 k_{x} b_{5}, \\
\bar{L}_{b_{6}}= & 2 k_{y} b_{6} .
\end{aligned}
$$

The variations of $\bar{L}$ with the coefficients of the free surface shape $\eta$ are

$$
\begin{aligned}
\bar{L}_{a_{1}}= & -4 k w a_{1} b_{3}-k w a_{3} b_{1}-k w a_{5} b_{1}-k w a_{6} b_{1}+6 k a_{1} b_{1}^{2} k_{x}^{2} \\
& +2 k a_{1} b_{1}^{2} k_{y}^{2}+2 k b_{1} k_{x} b_{5}-(9 / 4) w a_{1}^{2} b_{1} k^{2}-4 w a_{1} k_{x} b_{5} \\
& -2 w b_{1}+2 a_{1}+4 b_{1} b_{3} k^{2}+2 b_{1} k_{x}^{2} b_{5} \\
\bar{L}_{a_{3}}= & -k w a_{1} b_{1}-4 w b_{3}+2 a_{3}, \\
\bar{L}_{a_{5}}= & -k w a_{1} b_{1}-2 w b_{5}+a_{5}-b_{1}^{2} k_{y}^{2}, \\
\bar{L}_{a_{6}}= & -k w a_{1} b_{1}+a_{6}+b_{1}^{2} k_{x}^{2} .
\end{aligned}
$$

The variations of $\bar{L}$ with the wavenumber involve

$$
\begin{aligned}
\bar{L}_{k}= & -2 w a_{1}^{2} b_{3}-w a_{1} a_{3} b_{1}-w a_{1} a_{5} b_{1}-w a_{1} a_{6} b_{1} \\
& +3 a_{1}^{2} b_{1}^{2} k_{x}^{2}+a_{1}^{2} b_{1}^{2} k_{y}^{2}+2 a_{1} b_{1} k_{x} b_{5}+b_{1}^{2}+2 b_{3}^{2}, \\
& -(3 / 2) w a_{1}^{3} b_{1} k+8 a_{1} b_{1} b_{3} k \\
\partial \bar{L} / \partial k_{x}= & 6 k a_{1}^{2} b_{1}^{2} k_{x}+2 k a_{1} b_{1} b_{5}-2 w a_{1}^{2} b_{5}+4 a_{1} b_{1} k_{x} b_{5} \\
& +2 a_{6} b_{1}^{2} k_{x}+b_{5}^{2}, \\
\partial \bar{L} / \partial k_{y}= & 2 k a_{1}^{2} b_{1}^{2} k_{y}-2 a_{5} b_{1}^{2} k_{y}+b_{6}^{2} .
\end{aligned}
$$

where

$$
\begin{aligned}
\bar{L}_{k_{x}} & =\bar{L}_{k} k_{x} / k+\partial \bar{L} / \partial k_{x}, \\
\bar{L}_{k_{y}} & =\bar{L}_{k} k_{y} / k+\partial \bar{L} / \partial k_{y} .
\end{aligned}
$$




\section{References}

[1] T. C. Chen, "Experimental study on the solitary wave reflection along a straight sloped wall at oblique angle of incidence", U.S. Beach Erosion board Tech. Memo. 124, 1961.

[2] E. D. Cokelet, "Steep gravity waves in water of arbitrary uniform depth", Phil. Trans. R. Soc. Lond. A 286 (1977) 183-230.

[3] L. N. Howard and N. Kopell, "Slowly varying waves and shock structures in reactiondiffusion equations", Stud. Appl. Math. 56 (1977) 95-145.

[4] J. T. Kirby and R. A. Dalrymple, "A parabolic equation for the combined refractiondiffraction of Stokes" wave by mildiy varying topugraphy", j. Fiuid Míchi. 136 (1383) 453-466.

[5] J. C. Luke, “A variational principle for a fluid with a free surface", J. Fluid Mech. 27 (1967) 395-397.

[6] T. R. Marchant and A. J. Roberts, "Properties of short-crested waves in water of finite depth", J. Aust. Math. Soc. B 29 (1987) 103-125.

[7] T. R. Marchant and A. J. Roberts, “A variational approach to the problem of deep-water waves forming a circular caustic", J. Fluid Mech. 94 (1988) 581-597.

[8] C. C. Mei and E. O. Tuck, "Forward scattering by long thin bodies", SIAM J. Appl. Maths. 39 (1980) 178-191.

[9] D. H. Peregrine, "Wave jumps and caustics in the propagation of finite amplitude water waves", J. Fluid Mech. 136 (1983) 435-452.

[10] D. H. Peregrine, "Review Lecture", Proc. R. Soc. Lond. A 400 (1985) 1-18.

[11] P. H. Perroud, "The solitary wave reflection along a straight vertical wall at oblique angle”, Univ. of California, Berkeley, IER Tech. Rep. 99-3, 1957.

[12] A. J. Roberts, "Highly nonlinear short-crested waves", J. Fluid Mech. 135 (1983) 301321.

[13] A. J. Roberts and D. H. Peregrine, "Notes on long-crested water waves", J. Fluid Mech. 135 (1983) 323-335.

[14] T-K. Tsay and P. L.-F. Lui, "Numerical solutions of water-wave refraction and diffraction problems in the parabolic limit", J. Geophys. Res. 87 (1982) 7932-7940.

[15] G. B. Whitham, "Non-Linear dispersive waves", Proc. Roy. Soc. Lond A 283 (1965) 238-261.

[16] G. B. Whitham, Linear and Nonlinear Waves (Wiley-Interscience, 1974).

[17] R. L. Wiegel, "Water wave equivalent of Mach reflection", Proc. 9th Conf. Coastal Engng. A.S.C.E. (1964) 82-102.

[18] D. K. P. Yue and C. C. Mei, "Forward diffraction of Stokes waves by a thin wedge", $J$. Fluid Mech. 99 (1980) 33-52. 